\title{
A Mathematical Theory of Gravitational Collapse
}

\author{
Demetrios Christodoulou *
}

Departments of Mathematics and Physics, Syracuse University, Syracuse, NY 13210, USA

\begin{abstract}
We study the asymptotic behaviour, as the retarded time $u$ tends to infinity, of the solutions of Einstein's equations in the spherically symmetric case with a massless scalar field as the material model. We prove that when the final Bondi mass $M_{1}$ is different from zero, as $u \rightarrow \infty$, a black hole forms of mass $M_{1}$ surrounded by vacuum. We find the rate of decay of the metric functions and the behaviour of the scalar field on the horizon.
\end{abstract}

\section{Introduction}

In [1] we began the study of the global initial value problem for Einstein's equations $R_{\mu \nu}=8 \pi \partial_{\mu} \phi \partial_{\nu} \phi$ in the spherically symmetric case with a massless scalar field $\phi$ as the material model. Using a retarded time coordinate $r$, the spacetime metric can be put in the form

$$
d s^{2}=-e^{2 v} d u^{2}-2 e^{v+\lambda} d u d r+r^{2} d \Sigma^{2},
$$

where $d \Sigma^{2}$ is the metric of the standard 2-sphere. The problem is formulated most simply in terms of the function $h:=\partial(r \phi) / \partial r$. We define

$$
g:=\exp \left[-4 \pi \int_{r}^{\infty}(h-\bar{h})^{2} \frac{d r}{r}\right], \quad D:=\frac{\partial}{\partial u}-\frac{1}{2} \bar{g} \frac{\partial}{\partial r},
$$

and

$$
m:=\frac{r}{2}\left(1-\frac{\bar{g}}{g}\right), \quad \xi:=2 r D \bar{h},
$$

where, if $f$ is a function of $u$ and $r$, we denote by $\bar{f}$ the mean value function of $f$ :

$$
\bar{f}(u, r):=\frac{1}{r} \int_{0}^{r} f\left(u, r^{\prime}\right) d r^{\prime} .
$$

\footnotetext{
* Research supported in part by National Science Foundation grants MCS-8201599 to the Courant Institute and PHY-8318350 to Syracuse University
} 
We showed in [1] that the spherically symmetric Einstein-scalar equations are then equivalent to the equations

$$
D h=\frac{1}{2 r}(g-\bar{g})(h-\bar{h}), \quad \text { and } \quad D m=-\frac{\pi}{g} \xi^{2},
$$

through the identification $e^{v+\lambda}:=g, e^{v-\lambda}:=\bar{g}$ and $\phi:=\bar{h}$. The function $m(u, r)$ is the mass which at retarded time $u$ is enclosed within the sphere of radius $r$ and $M(u):=\lim _{r \rightarrow \infty} m(u, r)$ is the total (Bondi) mass at retarded time $u$. The integral curves of $D$, which we call characteristics, are the incoming light rays. The initial data of the problem is the specification of the function $h$ at $u=0$. In [1] we proved that if the initial data is sufficiently small there exists a global classical solution which disperses in the infinite future and the final Bondi Mass $M_{1}$ vanishes (Theorem 3 of [1]). In [2] we studied the problem in the large. We introduced there the concept of a generalized solution and we proved, without any restriction on the size of the initial data, the global existence of generalized solutions (Theorem 1 of [2]). In [3] we studied the regularity properties of generalized solutions and we proved a uniqueness theorem which shows that a generalized solution is an extension of a classical solution (Theorem 1 of [3]).

In the present paper we study the asymptotic behaviour of the generalized solutions as the retarted time $u$ tends to infinity. In the generic case for large initial data the final Bondi mass $M_{1}$ is different from zero. When $M_{1}=0$, as for the small solutions described by Theorem 3 of [1], the scalar field disperses and the spacetime tends to the Minkowski spacetime as $u \rightarrow \infty$. In the present paper we show that when $M_{1} \neq 0$, as $u \rightarrow \infty$, a black hole forms of mass $M_{1}$ surrounded by vacuum.

We shall assume, as in the previous papers, that the initial data which is given at $u=0$, satisfies the falloff conditions

$$
h(0, r)=O\left(r^{-3}\right) \quad \text { and } \quad \partial h / \partial r(0, r)=O\left(r^{-4}\right) \quad \text { as } \quad r \rightarrow \infty, \quad h:=\partial(r \phi) / \partial r .
$$

These conditions are not necessary. All the results of the present paper can be derived by the same methods under the weaker falloff conditions

$$
h(0, r)=O\left(r^{-1-\varepsilon}\right) \text { and } \partial h / \partial r(0, r)=O\left(r^{-2-\varepsilon}\right), \varepsilon>0 \quad \text { as } \quad r \rightarrow \infty .
$$

However, assuming only that the initial Bondi mass $M_{0}$ is finite is not sufficient to derive the results. The conditions $(*)$ are needed to ensure that the function $N:=\lim _{r \rightarrow \infty}(r \phi)$ is well defined.

The plan of the present paper is the following: In Sect. 1 we prove that in the region exterior to the Schwarzschild sphere $r=2 M_{1}$ corresponding to the final Bondi mass $M_{1}$, the solutions tend to stationarity in the sense that $\partial h / \partial u$ tends to zero as $u$ tends to infinity. The method of this section is not particular to the problem at hand and should apply also to other problems involving nonlinear evolution equations of the hyperbolic type (describing radiating physical systems). In Sect. 2 we prove that the mass remaining outside the sphere $r=2 M_{1}$ tends to zero as $u$ tends to infinity. The main part of this proof is the demonstration of the fact that $N \rightarrow 0$ as $u \rightarrow \infty$. In Sect. 3 we prove that an event horizon forms in the limit 
$u \rightarrow \infty$; the event horizon is the part of the limiting hypersurface $u=\infty$ interior to the sphere $r=2 M_{1}$. We derive the rate of decay of the metric functions and the asymptotic behaviour of the incoming light rays. The method of this section is based on an identity related to the scaling group covariance of the problem. In Sect. 4 we study the behaviour of the scalar field on the horizon and we derive some additional results about the global properties of the solutions.

\section{Asymptotic Stationarity Outside the Schwarzschild Radius}

The aim of this and the next section is the proof of:

Theorem 1. For $r>2 M_{1}, M(u)-m(u, r) \rightarrow 0$ as $u \rightarrow \infty$.

In this section we shall show that at each $r>2 M_{1}, \partial h / \partial u \rightarrow 0$ as $u \rightarrow \infty$. We start by deriving uniform bounds for $\bar{h}, h$ and $\partial h / \partial r$ in the region $r>r_{0}$, where $r_{0}$ is a constant greater than $2 M_{1}$. Let us recall the function $N$ defined in Sect. 2 of [3]:

$$
N:=\int_{0}^{\infty} h d r .
$$

Lemma 1. For each $r_{0}>2 M_{1}$ there are constants $C$ and $C^{\prime}$ (independent of $u$ ) such that

$$
\sup _{r \geqq r_{0}} r^{2}|h(u, r)| \leqq C, \text { and } \sup _{r \geqq r_{0}} r^{3}\left|\frac{\partial h}{\partial r}(u, r)\right| \leqq C^{\prime} .
$$

Also, $N$ is uniformly bounded: $|N| \leqq B$.

Proof. We first note that in the region $r \geqq r_{0}, \bar{g}$ has a positive lower bound (see Proposition 1 of [1]): according to (2.4) of [3],

$$
\bar{g}=1-\frac{2 M}{r}+\frac{1}{r} \int_{r}^{\infty}(1-g) d r,
$$

therefore

$$
\bar{g}\left(u, r_{0}\right) \geqq 1-\frac{2 M(u)}{r_{0}} .
$$

Since $M(u)$ is a monotonically nonincreasing function of $u$ tending to $M_{1}$ as $u \rightarrow \infty$, and we have $r_{0}>2 M_{1}$, there exists a $u_{1}$ such that for all $u \geqq u_{1}$,

$$
M(u) \leqq \frac{1}{2}\left(\frac{r_{0}}{2}+M_{1}\right),
$$

and therefore

$$
\bar{g}\left(u, r_{0}\right) \geqq \frac{1}{2}\left(1-\frac{2 M_{1}}{r_{0}}\right)^{2}>0 .
$$

On the other hand at each $r>0, \bar{g}(u, r)$, being a positive continuous function of $u$, has a positive minimum in the compact interval $\left[0, u_{1}\right]$. Hence $\bar{g}\left(u, r_{0}\right)$ has a positive infimum $k$, and therefore for all $r \geqq r_{0}$ and all $u \geqq 0$ we have

$$
\bar{g}(u, r) \geqq k>0 \text {. }
$$


This implies that in any region $r \geqq r_{0}, r_{0}>2 M_{1}$, the slope- $d u / d r$ of the characteristics has an upper bound, a fact which will be used repeatedly in the sequel.

Let us recall the fact that for any function $\left.f \in C^{1}\right] 0, \infty[$ such that $f$ and $r \partial f / \partial r$ belong to $L^{2}(0, \infty)$ and

$$
\lim _{r \rightarrow \infty} r f^{2}(r)=0
$$

it holds

$$
r f^{2} \leqq \int_{r}^{\infty} r^{2}\left(\frac{\partial f}{\partial r}\right)^{2} d r
$$

Applying this to the function $\bar{h}$ at each $u$, we obtain, in view of the positive lower bound for $\bar{g}$, that for all $r \geqq r_{0}>2 M_{1}$,

$$
r \bar{h}^{2} \leqq \int_{r}^{\infty}(h-\bar{h})^{2} d r \leqq \frac{1}{k} \int_{r}^{\infty} \frac{\bar{g}}{g}(h-\bar{h})^{2} d r \leqq \frac{M}{2 \pi k} .
$$

Therefore, in the region $r \geqq r_{0}$,

$$
|\bar{h}| \leqq\left(\frac{M_{0}}{2 \pi k}\right)^{1 / 2} \cdot \frac{1}{r^{1 / 2}}
$$

Integration of the nonlinear evolution equation along the characteristics $\chi$ gives:

$$
\begin{aligned}
h\left(u_{1}, r_{1}\right)= & \exp \left[\int_{0}^{u_{1}}\left[\frac{(g-\bar{g})}{2 r}\right]_{\chi} d u\right]\left\{h_{0}\left(\chi_{u_{1}}\left(0 ; r_{1}\right)\right)\right. \\
& \left.-\int_{0}^{u_{1}}\left[\frac{(g-\bar{g})}{2 r} \bar{h}\right]_{\chi} \exp \left[-\int_{0}^{u}\left[\frac{(g-\bar{g})}{2 r}\right]_{\chi} d u\right] d u\right\},
\end{aligned}
$$

where $\chi$ or $\chi_{u_{1}}\left(\cdot ; r_{1}\right)$ denotes the characteristic through $r=r_{1}$ at $u=u_{1}$. Since

$$
\frac{g-\bar{g}}{2 r}=\frac{m}{r^{2}} g \leqq \frac{M_{0}}{r^{2}}
$$

taking $r_{1} \geqq r_{0}>2 M_{1}$ and changing the variable integration along $\chi$ from $u$ to $r$, we can estimate:

$$
\begin{aligned}
\int_{0}^{u_{1}}\left[\frac{(g-\bar{g})}{2 r}\right]_{\chi} d u & \leqq M_{0} \int_{0}^{u_{1}} \frac{d u}{\left(\chi_{u_{1}}\left(u ; r_{1}\right)^{2}\right.} \\
& =2 M_{0} \int_{r_{1}}^{\chi_{u_{1}}\left(0 ; r_{1}\right)}\left[\frac{1}{\bar{g}}\right]_{\chi} \frac{d r}{r^{2}} \leqq \frac{2 M_{0}}{k} \int_{r_{1}}^{\chi_{u_{1}}\left(0 ; r_{1}\right)} \frac{d r}{r^{2}} \leqq \frac{2 M_{0}}{k} \cdot \frac{1}{r_{1}},
\end{aligned}
$$

using the upper bound $2 / k$ for the slope of the characteristics in the region $r \geqq r_{0}>2 M_{1}$. Taking into account (1.3) we can also estimate:

$$
\begin{aligned}
\int_{0}^{u_{1}} & {\left[\frac{(g-\bar{g})}{2 r}|\bar{h}|\right]_{\chi} d u \leqq \frac{M_{0}^{3 / 2}}{(2 \pi k)^{1 / 2}} \int_{0}^{u_{1}} \frac{d u}{\left(\chi_{u_{1}}\left(u ; r_{1}\right)\right)^{5 / 2}} } \\
& =\left(\frac{2}{\pi k}\right)^{1 / 2} M_{0}^{3 / 2} \int_{r_{1}}^{\chi_{u_{1}}\left(0 ; r_{1}\right)}\left[\frac{1}{\bar{g}}\right]_{\chi} \frac{d r}{r^{5 / 2}} \leqq\left(\frac{2}{\pi}\right)^{1 / 2}\left(\frac{M_{0}}{k}\right)^{3 / 2} \int_{r_{1}}^{\chi_{u_{1}}\left(0 ; r_{1}\right)} \frac{d r}{r^{5 / 2}} \\
& \leqq 3\left(\frac{1}{2 \pi}\right)^{1 / 2}\left(\frac{M_{0}}{k}\right)^{3 / 2} \cdot \frac{1}{r_{1}^{3 / 2}} .
\end{aligned}
$$


Setting then

$$
d_{0}:=\sup _{r \geqq 0}\left\{\left(r^{3}\left|h_{0}(r)\right|\right\},\right.
$$

we obtain from (1.4), in view of (1.6) and (1.7), that for all $u_{1} \geqq 0$ and all $r_{1} \geqq r_{0}$, $r_{0}>2 M_{1}$,

$$
\left|h\left(u_{1}, r_{1}\right)\right| \leqq e^{2 M_{0} / k r_{1}}\left[d_{0} \cdot \frac{1}{r_{1}^{3}}+3\left(\frac{1}{2 \pi}\right)^{1 / 2}\left(\frac{M_{0}}{k}\right)^{3 / 2} \cdot \frac{1}{r_{1}^{3 / 2}}\right]
$$

holds. Therefore, for each $r_{0}>2 M_{1}$ there exists a constant $C_{0}$ such that for all $u \geqq 0$ and $r \geqq r_{0}$,

$$
|h(u, r)| \leqq C_{0} / r^{3 / 2}
$$

Since

$$
N=r_{0} \bar{h}\left(r_{0}\right)+\int_{r_{0}}^{\infty} h d r
$$

and by (1.3),

$$
\left|\bar{h}\left(r_{0}\right)\right| \leqq\left(M_{0} / 2 \pi k r_{0}\right)^{1 / 2}
$$

(1.9) implies that $|N| \leqq B$, where

Since for $r \geqq r_{0}$,

$$
B:=\left(\frac{M_{0} r_{0}}{2 \pi k}\right)^{1 / 2}+\frac{2 C_{0}}{r_{0}^{1 / 2}} .
$$

$$
\bar{h}(r)=\frac{r_{0}}{r} \bar{h}\left(r_{0}\right)+\frac{1}{r} \int_{r_{0}}^{r} h d r,
$$

(1.9) also implies that in the region $r \geqq r_{0}$,

$$
|\bar{h}| \leqq B / r \text {. }
$$

Using (1.10) instead of (1.13) we now estimate

$$
\int_{0}^{u_{1}}\left[\frac{(g-\bar{g})}{2 r}|\bar{h}|\right]_{\chi} d u \leqq M_{0} B \int_{0}^{u_{1}} \frac{d u}{\left(\chi_{u_{1}}\left(u ; r_{1}\right)\right)^{3}} \leqq \frac{2 M_{0} B}{k} \int_{r_{1}}^{\chi u_{1}\left(0 ; r_{1}\right)} \frac{d r}{r^{3}} \leqq \frac{M_{0} B}{k} \cdot \frac{1}{r_{1}^{2}} .
$$

We then obtain from (1.4) that for all $u_{1} \geqq 0$ and all $r_{1} \geqq r_{0}$ :

$$
\left|h\left(u_{1}, r_{1}\right)\right| \leqq e^{2 M_{0} / k r_{1}}\left(d_{0} \cdot \frac{1}{r_{1}^{3}}+\frac{M_{0} B}{k} \cdot \frac{1}{r_{1}^{2}}\right)
$$

holds. Therefore, for each $r_{0}>2 M_{1}$ there exists a constant $C$ such that for all $u \geqq 0$ and $r \geqq r_{0}$,

$$
|h(u, r)| \leqq C / r^{2} .
$$

The evolution law of $\partial h / \partial r$, derived from the nonlinear evolution equation, is given by:

$$
D\left(\frac{\partial h}{\partial r}\right)=\frac{(g-\bar{g})}{r} \frac{\partial h}{\partial r}+\frac{1}{2 r^{2}}\left(-3(g-\bar{g})+4 \pi g(h-\bar{h})^{2}\right)(h-\bar{h}) .
$$


Integrating this along the characteristics we obtain

$$
\begin{aligned}
& \frac{\partial h}{\partial r}\left(u_{1}, r_{1}\right)=\exp \left[\int_{0}^{u_{2}}\left[\frac{(g-\bar{g})}{r}\right]_{\chi} d u\right]\left\{\frac{\partial h_{0}}{\partial r}\left(\chi_{u_{1}}\left(0 ; r_{1}\right)\right)\right. \\
& \left.\quad+\int_{0}^{u_{1}}\left[\frac{1}{2 r^{2}}\left(-3(g-\bar{g})+4 \pi g(h-\bar{h})^{2}\right)(h-\bar{h})\right]_{\chi} \exp \left[-\int_{0}^{u}\left[\frac{(g-\bar{g})}{r}\right]_{\chi} d u\right] d u\right\} .
\end{aligned}
$$

By (1.5), (1.10), and (1.12), in the region $r \geqq r_{0}$,

$$
\frac{1}{2 r^{2}}\left|-3(g-\bar{g})+4 \pi g(h-\bar{h})^{2}\right||h-\bar{h}| \leqq \frac{L}{r^{4}}
$$

holds, where

$$
L:=\left[3 M_{0}+\frac{2 \pi}{r_{0}}\left(\frac{C}{r_{0}}+B\right)^{2}\right]\left(\frac{C}{r_{0}}+B\right) .
$$

Taking $r_{1} \geqq r_{0}$ we can then estimate:

$$
\begin{aligned}
& \int_{0}^{u_{1}}\left[\frac{1}{2 r^{2}}\left|-3(g-\bar{g})+4 \pi g(h-\bar{h})^{2}\right||h-\bar{h}|\right]_{\chi} d u \\
& \quad \leqq L \int_{0}^{u_{1}} \frac{d u}{\left(\chi_{u_{1}}\left(u ; r_{1}\right)\right)^{4}} \leqq 2 \frac{L}{k} \int_{r_{1}}^{\chi_{u_{1}}\left(0: r_{1}\right)} \frac{d r}{r^{4}} \leqq \frac{2 L}{3 k} \cdot \frac{1}{r_{1}^{3}} .
\end{aligned}
$$

Defining

$$
d_{1}:=\sup _{r \geqq 0}\left\{r^{4}\left|\frac{\partial h_{0}}{\partial r}(r)\right|\right\},
$$

we obtain from (1.14), in view of (1.6) and (1.15), that for all $u_{1} \geqq 0$ and $r_{1} \geqq r_{0}$, $r_{0}>2 M_{1}$

$$
\left|\frac{\partial h}{\partial r}\left(u_{1}, r_{1}\right)\right| \leqq e^{4 M_{0} k r_{1}}\left(d_{1} \cdot \frac{1}{r_{1}^{4}}+\frac{2 L}{3 k} \cdot \frac{1}{r_{1}^{3}}\right)
$$

holds. Therefore, for each $r_{0}>2 M_{1}$ there exists a constant $C^{\prime}$ such that for all $u \geqq 0$ and $r \geqq r_{0}$,

$$
|\partial h / \partial r(u, r)| \leqq C^{\prime} / r^{3}
$$

Let us recall the total radiative amplitude $\Xi$ defined in Sect. 2 of [3]:

$$
\Xi:=\lim _{\delta \rightarrow 0} \int_{\delta}^{\infty} \bar{g}(h-\bar{h}) \frac{d r}{r} .
$$

The function $\Xi(u)$ is defined for almost all values of $u$ and we have $\Xi \in L^{2}(0, \infty)$. Let us set

$$
\eta\left(u_{0}\right):=\int_{u_{0}}^{\infty} \Xi^{2}(u) d u .
$$

Then $\eta\left(u_{0}\right) \rightarrow 0$ as $u_{0} \rightarrow \infty$. The quantity $\pi \eta\left(u_{0}\right)$ represents the total energy radiated after the retarded time $u_{0}$. The proof of asymptotic stationarity outside the final 
Schwarzschild radius uses the fact that the total energy radiated after the retarded time $u_{0}$ tends to zero as $u_{0}$ tends to infinity.

Lemma 2. For each $r_{0}>2 M_{1}$ and $\varepsilon>0$,

$$
\sup _{r \geqq r_{0}}\left\{r^{3-\varepsilon}\left|\frac{\partial h}{\partial u}(u, r)\right|\right\} \rightarrow 0 \quad \text { as } \quad u \rightarrow \infty .
$$

Proof. We consider the evolution law of $\partial h / \partial u$ along the characteristics:

$$
D\left(\frac{\partial h}{\partial u}\right)=\frac{1}{2} \frac{\partial \bar{g}}{\partial u} \frac{\partial h}{\partial r}+\frac{1}{2 r}\left(\frac{\partial g}{\partial u}-\frac{\partial \bar{g}}{\partial u}\right)(h-\bar{h})+\frac{1}{2 r}(g-\bar{g})\left(\frac{\partial h}{\partial u}-\frac{\partial \bar{h}}{\partial u}\right) .
$$

This law is obtained by differentiating the nonlinear evolution equation with respect to $u$. The idea now is to express the functions $\partial \bar{h} / \partial u, \partial g / \partial u$ and $\partial \bar{g} / \partial u$, which appear in the right-hand side of (1.18), at each point $(u, r)$ in terms of the total radiative amplitude $\Xi$ and the function $\partial h / \partial u$ at that value of $u$ from $r$ to $\infty$. Since

$$
\bar{h}=\frac{N}{r}-\frac{1}{r} \int_{r}^{\infty} h d r
$$

and, according to (2.10) of [3],

$$
\frac{\partial N}{\partial u}=\frac{1}{2} \Xi
$$

we have

$$
\frac{\partial \bar{h}}{\partial u}=\frac{\Xi}{2 r}-\frac{1}{r} \int_{r}^{\infty} \frac{\partial h}{\partial u} d r
$$

Since

$$
A=\int_{r}^{\infty}\left(h-\bar{h}^{2} \frac{d r}{r}, \quad \frac{\partial A}{\partial u}=2 \int_{r}^{\infty}(h-\bar{h})\left(\frac{\partial h}{\partial u}-\frac{\partial \bar{h}}{\partial u}\right) \frac{d r}{r},\right.
$$

and since $g=e^{-4 \pi A}, \partial g / \partial u=-4 \pi g \partial A / \partial u$. Also, according to (2.4) of [3],

$$
\bar{g}=1-\frac{2 M}{r}+\frac{1}{r} \int_{r}^{\infty}(1-g) d r,
$$

and according to (2.12) of [3],

$$
\frac{\partial M}{\partial u}=-\pi \Xi^{2}
$$

Therefore

$$
\frac{\partial \bar{g}}{\partial u}=\frac{2 \pi \Xi^{2}}{r}-\frac{1}{r} \int_{r}^{\infty} \frac{\partial g}{\partial u} d r
$$

Let now $\chi_{u_{1}}\left(\cdot ; r_{0}\right)$ denote the characteristic through $r=r_{0}$ at $u=u_{1}$, where $r_{0}$ is fixed and greater than $2 M_{1}$. For each $u \in\left[0, u_{1}\right]$ we set:

$$
\beta_{u_{1}}(u):=\sup _{r \geqq \chi_{u_{1}}\left(u ; r_{0}\right)}\left\{r^{2}\left|\frac{\partial h}{\partial u}(u, r)\right|\right\},
$$


where $\beta_{u_{1}}$ is a continuous function on $\left[0, u_{1}\right]$. By (1.21), for each $u \in\left[0, u_{1}\right]$ and $r \geqq \chi_{u_{1}}\left(u ; r_{0}\right)$, we have

$$
\begin{aligned}
\left|\frac{\partial \bar{h}}{\partial u}(u, r)\right| & \leqq \frac{|\Xi(u)|}{2 r}+\frac{1}{r} \int_{r}^{\infty}\left|\frac{\partial h}{\partial u}\left(u, r^{\prime}\right)\right| d r^{\prime} \\
& \leqq \frac{|\Xi(u)|}{2 r}+\frac{1}{r} \int_{r}^{\infty} \beta_{u_{1}}(u) \frac{d r^{\prime}}{r^{\prime 2}}=\frac{|\Xi(u)|}{2 r}+\frac{\beta_{u_{1}}(u)}{r^{2}} .
\end{aligned}
$$

By Lemma 1, in the region $r \geqq r_{0},|h-\bar{h}| \leqq C / r$ holds. Here and in the following $C$ shall denote various positive constants depending only on $r_{0}$. Considering the above we obtain from (1.22) that for $u \in\left[0, u_{1}\right]$ and $r \geqq \chi_{u_{1}}\left(u ; r_{0}\right)$,

$$
\begin{aligned}
\left|\frac{\partial A}{\partial u}(u, r)\right| & \leqq 2 \int_{r}^{\infty}|h-\bar{h}| \frac{\partial h}{\partial u}-\frac{\partial \bar{h}}{\partial u} \mid \frac{d r}{r} \\
& \leqq 2 \int_{r}^{\infty} C\left(\frac{|\Xi(u)|}{2 r^{\prime 3}}+\frac{2 \beta_{u_{1}}(u)}{r^{\prime 4}}\right) d r^{\prime}=C\left(\frac{|\Xi(u)|}{2 r^{2}}+\frac{4 \beta_{u_{1}}(u)}{3 r^{3}}\right),
\end{aligned}
$$

and therefore

$$
\left|\frac{\partial g}{\partial u}(u, r)\right| \leqq C\left(\frac{|\Xi(u)|}{r^{2}}+\frac{\beta_{u_{1}}(u)}{r^{3}}\right) .
$$

It then follows, in view of (1.25), that for each $u \in\left[0, u_{1}\right]$ and $r \geqq \chi_{u_{1}}\left(u ; r_{0}\right)$,

$$
\left|\frac{\partial \bar{g}}{\partial u}(u, r)\right| \leqq 2 \pi \frac{\Xi^{2}(u)}{r}+C^{\prime}\left(\frac{|\Xi(u)|}{r^{2}}+\frac{\beta_{u_{1}}(u)}{r^{3}}\right) \text {. }
$$

Taking into account (1.27), (1.28), and (1.29) as well as Lemma 1 and (1.5), we conclude, in view of (1.18), that in the region $\left\{(u, r) \mid 0 \leqq u \leqq u_{1}, r \geqq \chi_{u_{1}}\left(u ; r_{0}\right)\right\}$,

$$
\left|D\left(\frac{\partial h}{\partial u}\right)\right| \leqq C\left(\frac{\beta_{u_{1}}}{r^{4}}+\frac{|\Xi|}{r^{3}}+\frac{\Xi^{2}}{r^{3}}\right)
$$

holds, where $C$ is a constant depending on $r_{0}$ but not on $u_{1}$.

Let $\chi_{u_{1}}\left(\cdot ; r_{1}\right)$ denote the characteristic through $r=r_{1}$ at $u=u_{1}$, where $r_{1} \geqq r_{0}$. Integrating inequality (1.30) along such a characteristic we obtain:

$$
\begin{aligned}
\left|\frac{\partial h}{\partial u}\left(u, \chi_{u_{1}}\left(u ; r_{1}\right)\right)\right| \leqq & \left|\frac{\partial h}{\partial u}\left(u_{0}, \chi_{u_{1}}\left(u_{0} ; r_{1}\right)\right)\right| \\
& +C \int_{u_{0}}^{u} \frac{\beta_{u_{1}}\left(u^{\prime}\right)}{\left(\chi_{u_{1}}\left(u^{\prime} ; r_{1}\right)\right)^{4}} d u^{\prime}+C \int_{u_{0}}^{u} \frac{\left|\Xi\left(u^{\prime}\right)\right|+\Xi^{2}\left(u^{\prime}\right)}{\left(\chi_{u_{1}}\left(u^{\prime} ; r_{1}\right)\right)^{3}} d u^{\prime} .
\end{aligned}
$$

We shall multiply inequality $(1.31)$ by $\left(\chi_{u_{1}}\left(u ; r_{1}\right)\right)^{2}$, and we shall bound the right side by a quantity which is independent of $r_{1}$. Since $\chi_{u_{1}}\left(u ; r_{1}\right) \leqq \chi_{u_{1}}\left(u_{0} ; r_{1}\right)$, we have

$$
\left(\chi_{u_{1}}\left(u ; r_{1}\right)\right)^{2}\left|\frac{\partial h}{\partial u}\left(u_{0}, \chi_{u_{1}}\left(u_{0} ; r_{1}\right)\right)\right| \leqq l\left(u_{1}, u_{0}\right),
$$

where

$$
l\left(u_{1}, u_{0}\right)=\sup _{r \geqq \chi_{u_{1}}\left(u_{0} ; r_{0}\right)}\left\{r^{2}\left|\frac{\partial h}{\partial u}\left(u_{0}, r\right)\right|\right\} .
$$


Also,

$$
\left(\chi_{u_{1}}\left(u ; r_{1}\right)\right)^{2} \int_{u_{0}}^{u} \frac{\beta_{u_{1}}\left(u^{\prime}\right)}{\left(\chi_{u_{1}}\left(u^{\prime} ; r_{1}\right)\right)^{4}} d u^{\prime} \leqq \int_{u_{0}}^{u} \frac{\beta_{u_{1}}\left(u^{\prime}\right)}{\left(\chi_{u_{1}}\left(u^{\prime} ; r_{1}\right)\right)^{2}} d u^{\prime} \leqq \int_{u_{0}}^{u} \frac{\beta_{u_{1}}\left(u^{\prime}\right)}{\left(\chi_{u_{1}}\left(u^{\prime} ; r_{0}\right)\right)^{2}} d u^{\prime}
$$

Using the Schwarz inequality we estimate:

$$
\begin{aligned}
& \left(\chi_{u_{1}}\left(u ; r_{1}\right)\right)^{2} \int_{u_{0}}^{u} \frac{\left|\Xi\left(u^{\prime}\right)\right|}{\left(\chi_{u_{1}}\left(u^{\prime} ; r_{1}\right)\right)^{3}} d u^{\prime} \leqq \int_{u_{0}}^{u} \frac{\left|\Xi\left(u^{\prime}\right)\right|}{\chi_{u_{1}}\left(u^{\prime} ; r_{1}\right)} d u^{\prime} \\
& \leqq \int_{u_{0}}^{u_{1}} \frac{\left|\Xi\left(u^{\prime}\right)\right|}{\chi_{u_{1}}\left(u^{\prime} ; r_{0}\right)} d u^{\prime} \leqq\left(\int_{u_{0}}^{u_{1}} \Xi^{2}\left(u^{\prime}\right) d u^{\prime}\right)^{1 / 2}\left(\int_{u_{0}}^{u_{1}} \frac{d u}{\left(\chi_{u_{1}}\left(u^{\prime} ; r_{0}\right)\right)^{2}}\right)^{1 / 2} .
\end{aligned}
$$

Now,

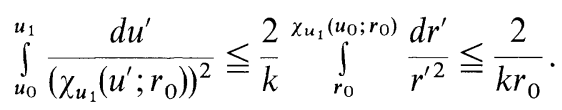

Hence (see (1.17)):

$$
\left(\chi_{u_{1}}\left(u ; r_{1}\right)\right)^{2} \int_{u_{0}}^{u} \frac{\left|\Xi\left(u^{\prime}\right)\right|}{\left(\chi_{u_{1}}\left(u^{\prime} ; r_{1}\right)\right)^{3}} d u \leqq C\left(\eta\left(u_{0}\right)\right)^{1 / 2} .
$$

We also estimate

$$
\left(\chi_{u_{1}}\left(u ; r_{1}\right)\right)^{2} \int_{u_{0}}^{u} \frac{\Xi^{2}\left(u^{\prime}\right)}{\left(\chi_{u_{1}}\left(u^{\prime} ; r_{1}\right)\right)^{3}} d u^{\prime} \leqq \int_{u_{0}}^{u_{1}} \frac{\Xi^{2}(u)}{\chi_{u_{1}}\left(u^{\prime} ; r_{0}\right)} d u^{\prime} \leqq \frac{1}{r_{0}} \cdot \eta\left(u_{0}\right) .
$$

Considering (1.33), (1.34), (1.36), and (1.37), we conclude from (1.31) that for all $u \in\left[0, u_{1}\right]$ :

$$
\begin{aligned}
\beta_{u_{1}}(u) & =\sup _{r_{1} \geqq r_{0}}\left\{\left(\chi_{u_{1}}\left(u ; r_{1}\right)\right)^{2}\left|\frac{\partial h}{\partial u}\left(u, \chi_{u_{1}}\left(u ; r_{1}\right)\right)\right|\right\} \\
& \leqq l\left(u_{1}, u_{0}\right)+C\left(\eta^{1 / 2}\left(u_{0}\right)+\eta\left(u_{0}\right)\right)+C \int_{u_{0}}^{u} \frac{\beta_{u_{1}}\left(u^{\prime}\right)}{\left(\chi_{u_{1}}\left(u^{\prime} ; r_{0}\right)\right)^{2}} d u^{\prime} .
\end{aligned}
$$

We have thus derived a linear integral inequality for $\beta_{u_{1}}$. We conclude that

$$
\beta_{u_{1}}(u) \leqq\left[l\left(u_{1}, u_{0}\right)+C\left(\eta^{1 / 2}\left(u_{0}\right)+\eta\left(u_{0}\right)\right)\right] \exp \left[C \int_{u_{0}}^{u} \frac{d u^{\prime}}{\left(\chi_{u_{1}}\left(u^{\prime} ; r_{0}\right)\right)^{2}}\right] .
$$

Therefore, in view of (1.35),

$$
\sup _{r \geqq r_{0}}\left\{r^{2}\left|\frac{\partial h}{\partial u}\left(u_{1}, r\right)\right|\right\}=\beta_{u_{1}}\left(u_{1}\right) \leqq C\left(l\left(u_{1}, u_{0}\right)+\eta^{1 / 2}\left(u_{0}\right)+\eta\left(u_{0}\right)\right),
$$

where $C$ is a constant depending on $r_{0}$ but independent of $u_{1}$ and $u_{0}$.

Lemma 1 implies through the nonlinear evolution equation that there exists a constant $C^{\prime \prime}$ depending only on $r_{0}$ such that for all $u \geqq 0$ and $r \geqq r_{0}$,

$$
\left|\frac{\partial h}{\partial u}(u, r)\right| \leqq \frac{C^{\prime \prime}}{r^{3}} .
$$


The upper bound of the slope of the characteristics in the region $r \geqq r_{0}$ implies that

$$
\chi_{u_{1}}\left(u ; r_{0}\right) \geqq r_{0}+\frac{k}{2}\left(u_{1}-u_{0}\right) .
$$

As a consequence of (1.40) and (1.41) [see (1.33)]:

$$
l\left(u_{1}, u_{0}\right) \leqq \frac{C^{\prime \prime}}{r_{0}+\frac{k}{2}\left(u_{1}-u_{0}\right)} .
$$

Given now any $\delta>0$, we first choose $u_{0}$ large enough so that

$$
\eta^{1 / 2}\left(u_{0}\right)+\eta\left(u_{0}\right) \leqq \delta / 2 C
$$

We then choose $u_{2}$ large enough so that

$$
r_{0}+\frac{k}{2}\left(u_{2}-u_{0}\right) \geqq \frac{2 C C^{\prime \prime}}{\delta}
$$

Then by (1.39), in view of (1.42), for all $u_{1}>u_{2}$ we have:

$$
\sup _{r \geqq r_{0}}\left\{r^{2}\left|\frac{\partial h}{\partial u}(u, r)\right|\right\}<\delta \text {. }
$$

Therefore

$$
\sup _{r \geqq r_{0}}\left\{r^{2}\left|\frac{\partial h}{\partial u}(u, r)\right|\right\} \rightarrow 0 \text { as } u \rightarrow \infty .
$$

Finally, let $\varepsilon$ be any positive real number. By (1.40), for $r_{1} \geqq r_{0}$,

$$
\sup _{r \geqq r_{1}}\left\{r^{3-\varepsilon}\left|\frac{\partial h}{\partial u}(u, r)\right|\right\}<\frac{C^{\prime \prime}}{r_{1}^{c}} .
$$

Given any $\delta>0$, we first choose $r_{1}$ such that

$$
r_{1}>\left(C^{\prime \prime} / \delta\right)^{1 / \varepsilon}
$$

Then, according to (1.43), we can choose $u_{1}$ such that for all $u>u_{1}$ we have

$$
\sup _{r \geqq r_{0}}\left\{r^{2}\left|\frac{\partial h}{\partial u}(u, r)\right|\right\}<\frac{\delta}{r_{1}^{1-\varepsilon}} .
$$

As a consequence of (1.46) together with (1.44) and (1.45), for all $u>u_{1}$,

$$
\sup _{r \geqq r_{0}}\left\{r^{3-\varepsilon}\left|\frac{\partial h}{\partial u}(u, r)\right|\right\}<\delta
$$

holds. We conclude that

$$
\sup \left\{r^{3-\varepsilon}\left|\frac{\partial h}{\partial u}(u, r)\right|\right\} \rightarrow 0 \quad \text { as } \quad u \rightarrow \infty .
$$




\section{Asymptotic Tendency to Vacuum Outside the Schwarzschild Radius}

The proof of Theorem 1 relies on establishing first that $N(u) \rightarrow 0$ as $u \rightarrow \infty$. The proof of this, in turn, uses the following lemma:

Lemma 3. If, at a certain value of $u, N(u) \neq 0$, and for some $r_{1}>0$,

$$
\sup _{r \geqq r_{1}}\left\{r^{5 / 2}\left|\frac{\partial h}{\partial u}(u, r)\right|\right\} \leqq \frac{1}{8}|N(u)| r_{1}^{1 / 2}
$$

then at that value of $u$,

$$
\bar{g}\left(u, r_{1}\right) \geqq \frac{\pi}{2} \frac{N^{2}(u)}{M(u)} \frac{1}{r_{1}} .
$$

Proof. The nonlinear evolution equation can be written in the form

$$
\frac{\partial}{\partial r}(r \bar{g}(h-\bar{h}))=2 r \frac{\partial h}{\partial u} \text {. }
$$

Since at each $u r \bar{g}(h-\bar{h}) \rightarrow-N$ as $r \rightarrow \infty$, integrating (2.1) at the given value of $u$ from $r$ to $\infty$, we obtain

$$
r \bar{g}(h-\bar{h})+N=-2 \int_{r}^{\infty} r \frac{\partial h}{\partial u} d r .
$$

Since $N(u) \neq 0$, we can define

$$
f(u, r):=1+\frac{2}{N(u)} \int_{r}^{\infty} r^{\prime} \frac{\partial h}{\partial u}\left(u, r^{\prime}\right) d r^{\prime} .
$$

For $r \geqq r_{1}$ we have

$$
\left|\int_{r}^{\infty} r^{\prime} \frac{\partial h}{\partial u}\left(u, r^{\prime}\right) d r^{\prime}\right| \leqq \sup _{r \geqq r_{1}}\left\{r^{5 / 2}\left|\frac{\partial h}{\partial u}(u, r)\right|\right\} \int_{r}^{\infty} \frac{d r^{\prime}}{r^{\prime 3 / 2}} \leqq \frac{2}{r_{1}^{1 / 2}} \sup _{r \geqq r_{1}}\left\{r^{5 / 2}\left|\frac{\partial h}{\partial u}(u, r)\right|\right\},
$$

and therefore, by the assumption of the lemma,

$$
\left|\int_{r}^{\infty} r^{\prime} \frac{\partial h}{\partial u}\left(u, r^{\prime}\right) d r^{\prime}\right| \leqq \frac{1}{4}|N(u)|
$$

It then follows from (2.3) that

$$
\inf _{r \geqq r_{1}} f(u, r) \geqq \frac{1}{2} .
$$

Thus at the given value of $u$ we can define in the interval $\left[r_{1}, \infty[\right.$ the function $\theta$ :

$$
\theta:=\frac{(h-\bar{h})}{f} .
$$

According to (2.2) and (2.3),

$$
\theta=-\frac{N}{r \bar{g}}
$$


Differentiating this equation with respect to $r$ we obtain:

$$
\frac{\partial \theta}{\partial r}=\frac{N g}{r^{2} \bar{g}^{2}}=\frac{g}{N} \theta^{2}
$$

On the other hand,

$$
\frac{\partial g}{\partial r}=\frac{4 \pi}{r} g(h-\bar{h})^{2}=\frac{4 \pi}{r} g f^{2} \theta^{2}
$$

Consequently,

$$
\frac{\partial \theta}{\partial r}=\frac{1}{4 \pi f^{2} N} r \frac{\partial g}{\partial r} .
$$

Since $\theta \rightarrow 0$ for $r \rightarrow \infty$, integrating (2.8) from $r$ to $\infty$, we obtain that in the interval $\left[r_{1}, \infty[\right.$,

$$
\theta=-\frac{1}{4 \pi} \frac{1}{f^{2} N} \int_{r}^{\infty} r \frac{\partial g}{\partial r} d r
$$

Taking into account (2.5) and the fact that

$$
\int_{r}^{\infty} r \frac{\partial g}{\partial r} d r \leqq \int_{0}^{\infty} r \frac{\partial g}{\partial r} d r=\int_{0}^{\infty}(1-g) d r=2 M,
$$

we conclude from (2.9) that in the interval $\left[r_{1}, \infty[\right.$ :

$$
|\theta| \leqq \frac{2}{\pi} \frac{M}{|N|}
$$

Equation (2.7) then implies that for all $r \geqq r_{1}$,

$$
r \bar{g}(u, r) \geqq \frac{\pi}{2} \frac{N^{2}(u)}{M(u)} .
$$

We are now ready to demonstrate

Lemma 4. $N(u) \rightarrow 0$ as $u \rightarrow \infty$.

Proof. The proof will be by contradiction. Let us suppose that $N$ does not tend to zero as $u$ tends to infinity. Then there is an $\varepsilon>0$ and a sequence $\left\{u_{n}\right\}, u_{n} \rightarrow \infty$ for $n$ $\rightarrow \infty$, such that $\left|N\left(u_{n}\right)\right| \geqq 2 \varepsilon$. In view of the fact that $\partial N / \partial u=(1 / 2) \Xi$, for each $n$ and each $u \geqq u_{n}$, we then have

$$
|N(u)| \geqq 2 \varepsilon-\frac{1}{2} \int_{u_{n}}^{u}\left|\Xi\left(u^{\prime}\right)\right| d u^{\prime} \geqq 2 \varepsilon-\frac{1}{2}\left(u-u_{n}\right)^{1 / 2}\left(\int_{u_{n}}^{\infty} \Xi^{2}\left(u^{\prime}\right) d u^{\prime}\right)^{1 / 2} .
$$

Thus if we define $u_{n}^{\prime}$ by

$$
u_{n}^{\prime}-u_{n}=4 \varepsilon^{2} / \eta\left(u_{n}\right)
$$

[see (1.17)] we have a sequence of intervals $\left[u_{n}, u_{n}^{\prime}\right]$ of increasing length,

$$
u_{n}^{\prime}-u_{n} \rightarrow \infty \text { for } n \rightarrow \infty \text {, }
$$


and in each interval

$$
\inf _{u \in\left[u_{n}, u_{n}^{\prime}\right]}|N(u)| \geqq \varepsilon .
$$

We now define for each $i, i=0,1,2, \ldots$, a sequence of bands $A_{i, n}$,

$$
A_{i, n}:=\left[u_{i, n}, u_{n}^{\prime}\right] \times\left[r_{i}, \infty[.\right.
$$

The $r_{i}$ are defined recursively by:

$$
r_{i+1}=r_{i} e^{-\pi \varepsilon^{2} / 8 M_{1} r_{i}},
$$

starting from some $r_{0}>2 M_{1}$. We may, in fact, choose $r_{0}$ so that

$$
\frac{1}{2}\left(r_{1}+r_{0}\right)=2 M_{1} \text {. }
$$

Then, starting from $u_{0, n}:=u_{n}$, the $u_{i, n}$ are defined recursively by requiring that for each $n$ the points $\left(u_{i+1, n}, r_{i+1}\right)$ and $\left(u_{i, n}, r_{i}\right)$ lie on the same characteristic curve:

$$
\chi_{u_{i+1, n}}\left(u_{i, n} ; r_{i+1}\right)=r_{i} \text {. }
$$

We shall show in the following that, for each $i, u_{n}^{\prime}-u_{i, n} \rightarrow \infty$ for $n \rightarrow \infty$.

For any given $i$, let $P_{i}$ be the following proposition:

1) $u_{n}^{\prime}-u_{i, n} \rightarrow \infty$ for $n \rightarrow \infty$.

2) There are constants: $B_{i}, C_{i}, C_{i}^{\prime}$, such that for all $n$ large enough

$$
\sup _{A_{i, n}}\{r|\bar{h}|\} \leqq B_{i}, \quad \sup _{A_{i, n}}\left\{r^{2}|h|\right\} \leqq C_{i}, \quad \sup _{A_{i, n}}\left\{r^{3}\left|\frac{\partial h}{\partial r}\right|\right\} \leqq C_{i}^{\prime} .
$$

3) $\sup _{A_{i, n}}\left\{r^{5 / 2}\left|\frac{\partial h}{\partial u}\right|\right\} \rightarrow 0$ for $n \rightarrow \infty$.

Since $r_{0}>2 M_{1}$, according to Lemma 1 , for all $u$,

$$
\begin{gathered}
\sup _{r \geqq r_{0}}\{r|\bar{h}(u, r)|\} \leqq B, \\
\sup _{r \geqq r_{0}}\left\{r^{2}|h(u, r)|\right\} \leqq C, \\
\sup _{r \geqq r_{0}}\left\{r^{3}\left|\frac{\partial h}{\partial r}(u, r)\right|\right\} \leqq C^{\prime},
\end{gathered}
$$

holds, and by Lemma 2,

$$
\sup _{r \geqq r_{0}}\left\{r^{5 / 2}\left|\frac{\partial h}{\partial u}(u, r)\right|\right\} \rightarrow 0 \text { as } u \rightarrow \infty .
$$

It follows, in view of $(2.11)$, that the proposition $P_{0}$ is true. We shall now demonstrate that the proposition $P_{i}$ implies the proposition $P_{i+1}$. The proposition $P_{i}$ would then be true, by induction, for each $i$.

Let proposition $P_{i}$ hold for some $i$. Then by part 3) there exists a $N_{i}$ such that for all $n \geqq N_{i}$ :

$$
\sup _{A_{l, n}}\left\{r^{5 / 2}\left|\frac{\partial h}{\partial u}\right|\right\} \leqq \frac{1}{8} \varepsilon r_{i}^{1 / 2}
$$


As we can choose $N_{i}$ to satisfy also

$$
M\left(u_{N_{2}}\right) \leqq 2 M_{1} .
$$

Lemma 3 implies that for all $n \geqq N_{i}$ :

$$
\inf _{u \in\left[u_{i, n}, u_{n}^{\prime}\right]} \bar{g}\left(u, r_{i}\right) \geqq \frac{\pi \varepsilon^{2}}{4 M_{1}} \cdot \frac{1}{r_{i}} .
$$

As a consequence of the fact that $\partial \bar{g} / \partial r \leqq 1 / r$, we have

$$
\bar{g}\left(u, r_{i+1}\right) \geqq \bar{g}\left(u, r_{i}\right)-\log \left(\frac{r_{i}}{r_{i+1}}\right) .
$$

Hence (2.14) together with (2.17), imply that for $n \geqq N_{i}$,

$$
\inf _{u \in\left[u_{i, n}, u_{n}^{\prime}\right]} \bar{g}\left(u, r_{i+1}\right) \geqq \frac{\pi \varepsilon^{2}}{8 M_{1}} \cdot \frac{1}{r_{i}}:=k_{i} .
$$

By (2.19) in the regions $\left[u_{i, n}, u_{n}^{\prime}\right] \times\left[r_{i+1}, \infty[\right.$ the slope $-d u / d r$ of the characteristics has for $n \geqq N_{i}$ an upper bound $2 / k_{i}$ independent of $n$. Hence for all $n \geqq N_{i}$,

$$
u_{i+1, n}-u_{i, n} \leqq \frac{2}{k_{i}}\left(r_{i}-r_{i+1}\right)
$$

[see (2.16)]. It follows that part 1) of proposition $P_{i+1}$ holds.

From (2.19) we deduce in each $\left[u_{i, n}, u_{n}^{\prime}\right] \times\left[r_{i+1}, \infty\left[, n \geqq N_{i}\right.\right.$ (see proof of Lemma 1):

$$
r \bar{h}^{2} \leqq \int_{r}^{\infty}(h-\bar{h})^{2} d r \leqq \frac{1}{k_{i}} \int_{r}^{\infty} \frac{\bar{g}}{g}(h-\bar{h})^{2} d r \leqq \frac{M}{2 \pi k_{i}} \leqq \frac{M_{1}}{\pi k_{i}},
$$

and by part 2) of proposition $P_{i}$ there exists a constant $B_{i}$ such that for all $n$ large enough, $|\bar{h}| \leqq \frac{B_{i}}{r}$ in $\left[u_{i, n}, u_{n}^{\prime}\right] \times\left[r_{i}, \infty\left[\right.\right.$. We conclude that there exists a constant $B_{i+1}$ such that for all $n$ large enough:

$$
\sup _{\left[u_{i, n}, u_{n}^{\prime}\right] \times\left[r_{i}+1, \infty[\right.}\{r|\bar{h}|\} \leqq B_{i+1} .
$$

Consider the regions

$$
A_{i+1, n}^{\prime}:=\left\{(u, r) \mid r \geqq \chi_{u_{l+1, n}}\left(u ; r_{i+1}\right), u_{i, n} \leqq u \leqq u_{i+1, n}\right\} \cup A_{i+1, n} .
$$

We evidently have:

$$
A_{i+1, n} \subset A_{i+1, n}^{\prime} \subset\left[u_{i, n}, u_{n}^{\prime}\right] \times\left[r_{i+1}, \infty[.\right.
$$

For every $\left(u^{\prime}, r^{\prime}\right) \in A_{i+1, n}^{\prime}$, the segment of the characteristic $\chi$ through $\left(u^{\prime}, r^{\prime}\right)$ between $u_{i, n}$ and $u^{\prime}$ is contained in $A_{i+1, n}^{\prime}$ :

$$
\left\{\left(u, \chi_{u^{\prime}}\left(u ; r^{\prime}\right)\right)\left\{u \in\left[u_{i, n}, u^{\prime}\right]\right\} \subset A_{i+1, n}^{\prime} .\right.
$$

Integrating the nonlinear evolution equation along $\chi$ gives:

$$
\begin{aligned}
& h\left(u^{\prime}, r^{\prime}\right)=\exp \left[\int_{u_{t, n}}^{u^{\prime}}\left[\frac{(g-\bar{g})}{2 r}\right]_{\chi} d u\right]\left\{h\left(u_{i, n}, \chi_{u^{\prime}}\left(u_{i, n} ; r^{\prime}\right)\right)\right. \\
& \left.-\int_{u_{t, n}}^{u^{\prime}}\left[\frac{(g-\bar{g})}{2 r} \bar{h}\right]_{\chi} \exp \left[-\int_{u_{\imath, n}}^{u}\left[\frac{(g-\bar{g})}{2 r}\right]_{\chi} d u\right] d u\right\},
\end{aligned}
$$


where $\chi$ or $\chi_{u^{\prime}}\left(\cdot ; r^{\prime}\right)$ denotes the characteristic through $\left(u^{\prime}, r^{\prime}\right)$. Taking into account the fact that for $u \geqq u_{i, n}, n$ large enough,

$$
\frac{(g-\bar{g})}{2 r} \leqq \frac{M}{r^{2}} \leqq \frac{2 M_{1}}{r^{2}}
$$

we obtain, in view of (2.19),

$$
\begin{aligned}
\int_{u_{i, n}}^{u^{\prime}}\left[\frac{(g-\bar{g})}{2 r}\right]_{\chi} & \leqq 2 M_{1} \int_{u_{i, n}}^{u^{\prime}} \frac{d u}{\left(\chi_{u^{\prime}}\left(u ; r^{\prime}\right)\right)^{2}}=4 M_{1} \int_{r^{\prime}}^{\chi_{u^{\prime}}\left(u_{i, n} ; r^{\prime}\right)}\left[\frac{1}{\bar{g}}\right]_{\chi} \frac{d r}{r^{2}} \\
& \leqq \frac{4 M_{1}}{k_{i}} \int_{u^{\prime}\left(u_{i, n} ; r^{\prime}\right)}^{\frac{d r}{r^{2}} \leqq \frac{4 M_{1}}{k_{i}} \cdot \frac{1}{r^{\prime}},}
\end{aligned}
$$

and, in view of (2.20),

$$
\begin{aligned}
& \int_{u_{i, n}}^{u^{\prime}}\left[\frac{(g-\bar{g})}{2 r}|\bar{h}|\right]_{\chi} d u \leqq 2 M_{1} B_{i+1} \int_{u_{i, n}}^{u^{\prime}} \frac{d u}{\left(\chi_{u^{\prime}}\left(u ; r^{\prime}\right)\right)^{3}} \\
& \leqq \frac{4 M_{1} B_{i+1}}{k_{i}} \int_{r^{\prime}}^{\chi_{u^{\prime}}\left(u_{i, n} ; r^{\prime}\right)} \frac{d r}{r^{3}} \leqq \frac{2 M_{1} B_{i+1}}{k_{i}} \cdot \frac{1}{r^{\prime 2}} .
\end{aligned}
$$

Since for all $\left(u^{\prime}, r^{\prime}\right) \in A_{i+1, n}$ :

$$
\chi_{u^{\prime}}\left(u_{i, n} ; r^{\prime}\right) \geqq r_{i},
$$

part 2) of proposition $P_{i}$ implies that

$$
\left|h\left(u_{i, n}, \chi_{u^{\prime}}\left(u_{i, n} ; r^{\prime}\right)\right)\right| \leqq \frac{C_{i}}{\left(\chi_{u^{\prime}}\left(u_{i, n} ; r^{\prime}\right)\right)^{2}} \leqq \frac{C_{i}}{r^{\prime 2}}
$$

(if $n$ is large enough). In view of (2.25), (2.26), and (2.28) we conclude from (2.24) that for all $n$ large enough and all $\left(u^{\prime}, r^{\prime}\right) \in A_{i+1, n}^{\prime}$ :

$$
\left|h\left(u^{\prime}, r^{\prime}\right)\right| \leqq e^{4 M_{1} / k_{2} r^{\prime}}\left(C_{i}+\frac{2 M_{1}}{k_{i}} B_{i+1}\right) \frac{1}{r^{\prime 2}} .
$$

Therefore, there exists a constant $C_{i+1}$ such that for all $n$ large enough

$$
\sup _{\boldsymbol{A}_{\boldsymbol{i}+1, n}^{\prime}}\left\{\boldsymbol{r}^{2}|h|\right\} \leqq C_{i+1} \text {. }
$$

Taking again $\left(u^{\prime}, r^{\prime}\right) \in A_{i+1, n}^{\prime}$, and integrating (1.13) along the characteristic $\chi_{u^{\prime}}\left(\cdot ; r^{\prime}\right)$, we obtain

$$
\begin{gathered}
\frac{\partial h}{\partial r}\left(u^{\prime}, r^{\prime}\right)=\exp \left[\int_{u_{i, n}}^{u^{\prime}}\left[\frac{(g-\bar{g})}{r}\right]_{\chi} d u\right]\left\{\frac{\partial h}{\partial r}\left(u_{i, n}, \chi_{u^{\prime}}\left(u_{i, n} ; r^{\prime}\right)\right)\right. \\
\left.+\int_{u_{i, n}}^{u^{\prime}}\left[\frac{1}{2 r^{2}}\left(-3(g-\bar{g})+4 \pi g(h-\bar{h})^{2}\right)(h-\bar{h})\right]_{\chi} \exp \left[-\int_{u_{i, n}}^{u}\left[\frac{(g-\bar{g})}{r}\right]_{\chi} d u\right] d u\right\} .
\end{gathered}
$$

By (2.29) and (2.20) in $A_{i+1, n}^{\prime}$ for all $n$ large enough we have

$$
\frac{1}{2 r^{2}}\left|-3(g-\bar{g})+4 \pi g(h-\bar{h})^{2}\right||h-\bar{h}| \leqq \frac{L_{i+1}}{r^{4}},
$$


where

$$
L_{i+1}:=2\left[3 M_{1}+\frac{\pi}{r_{i+1}}\left(\frac{C_{i+1}}{r_{i+1}}+B_{i}\right)^{2}\right]\left(\frac{C_{i+1}}{\check{r}_{i+1}}+B_{i}\right) .
$$

Therefore, considering (2.23), we can estimate

$$
\begin{aligned}
& \int_{u_{i, n}}^{u^{\prime}}\left[\frac{1}{2 r^{2}}\left|-3(g-\bar{g})+4 \pi g(h-\bar{h})^{2}\right||h-\bar{h}|\right]_{\chi} d u \\
& \quad \leqq L_{i+1} \int_{u_{i, n}}^{u^{\prime}} \frac{d u}{\left(\chi_{u^{\prime}}\left(u ; r^{\prime}\right)\right)^{4}} \leqq \frac{2 L_{i+1}}{k_{i}} \int_{r^{\prime}}^{\chi_{u^{\prime}}\left(u_{i, n} ; r^{\prime}\right)} \frac{d r}{r^{4}} \leqq \frac{2 L_{i+1}}{3 k_{i}} \cdot \frac{1}{r^{\prime}},
\end{aligned}
$$

considering (2.27), part 2) of proposition $P_{i}$ implies that:

$$
\left|\frac{\partial h}{\partial r}\left(u_{i, n}, \chi_{u^{\prime}}\left(u_{i, n} ; r^{\prime}\right)\right)\right| \leqq \frac{C_{i}^{\prime}}{\left(\chi_{u^{\prime}}\left(u_{i, n} ; r^{\prime}\right)\right)^{3}} \leqq \frac{C_{i}^{\prime}}{r^{\prime 3}}
$$

(if $n$ is large enough). In view of (2.25), (2.31), and (2.32), we conclude from (2.30) that for all $n$ large enough and all $\left(u^{\prime}, r^{\prime}\right) \in A_{i+1, n}^{\prime}$ :

$$
\left|\frac{\partial h}{\partial r}\left(u^{\prime}, r^{\prime}\right)\right| \leqq e^{8 M_{1} / k_{k} r^{\prime}}\left(C_{i}^{\prime}+\frac{2 L_{i+1}}{3 k_{i}}\right) \frac{1}{r^{\prime 3}} .
$$

Therefore there exists a constant $C_{i+1}^{\prime}$ such that for all $n$ large enough:

$$
\sup _{A_{i+1, n}^{\prime}}\left\{r^{3}\left|\frac{\partial h}{\partial r}\right|\right\} \leqq C_{i+1}^{\prime} \text {. }
$$

Considering (2.22), (2.20) together with (2.29) and (2.33) imply that part 2) of proposition $P_{i+1}$ holds.

Now, for each $u^{\prime} \in\left[u_{i+1, n}, u_{n}^{\prime}\right]$ let $\chi_{u^{\prime}}\left(\cdot ; r_{i+1}\right)$ denote the characteristics through $\left(u^{\prime}, r_{i}\right)$. Then for every $u \in\left[u_{i, n}, u^{\prime}\right]$ the half-line $\left\{(u, r) \mid r \geqq \chi_{u^{\prime}}\left(u ; r_{i+1}\right)\right\}$ is contained in $A_{i+1, n}^{\prime}$. For each $u \in\left[u_{i, n}, u^{\prime}\right]$ we set:

$$
\beta_{u^{\prime}}(u):=\sup _{r \geqq \chi u^{\prime}\left(u ; r_{l}+1\right)}\left\{r^{2}\left|\frac{\partial h}{\partial u}(u, r)\right|\right\} .
$$

Then for each $u \in\left[u_{i, n}, u^{\prime}\right]$ and $r \geqq \chi_{u^{\prime}}\left(u ; r_{i+1}\right)$ we have (see proof of Lemma 2 ):

$$
\left|\frac{\partial \bar{h}}{\partial u}(u, r)\right| \leqq \frac{|\Xi(u)|}{2 r}+\frac{\beta_{u^{\prime}}(u)}{r^{2}},
$$

and using (2.20) and (2.29) we can deduce, as in the proof of Lemma 2, that

$$
\left|\frac{\partial g}{\partial u}(u, r)\right| \leqq C\left(\frac{|\Xi(u)|}{r^{2}}+\frac{\beta_{u^{\prime}}(u)}{r^{3}}\right),
$$

and

$$
\left|\frac{\partial \bar{g}}{\partial u}(u, r)\right| \leqq \frac{2 \pi \Xi^{2}(u)}{r}+C\left(\frac{|\Xi(u)|}{r^{2}}+\frac{\beta_{u^{\prime}}(u)}{r^{3}}\right)
$$


Here and in the following, $C$ shall denote various positive constants depending on $i$ but independent of $n$. Taking into account (2.35), (2.36), and (2.37) together with (2.20), (2.29), and (2.33), we conclude from (1.18) that in each region

$$
\left\{(u, r) \mid u_{i, n} \leqq u \leqq u^{\prime}, r \geqq \chi_{u^{\prime}}\left(u ; r_{i+1}\right)\right\}
$$

(n large enough),

$$
\left|D\left(\frac{\partial h}{\partial u}\right)\right| \leqq C\left(\frac{\beta_{u^{\prime}}}{r^{4}}+\frac{|\Xi|}{r^{3}}+\frac{\Xi^{2}}{r^{2}}\right)
$$

holds.

Let $\chi_{u^{\prime}}\left(\cdot ; r^{\prime}\right)$ denote the characteristic through $\left(u^{\prime}, r^{\prime}\right)$, where $r^{\prime} \geqq r_{i+1}$. Integrating inequality (2.38) along such a characteristic we obtain:

$$
\begin{aligned}
\left|\frac{\partial h}{\partial u}\left(u, \chi_{u^{\prime}}\left(u ; r^{\prime}\right)\right)\right| \leqq & \left|\frac{\partial h}{\partial u}\left(u_{i, n}, \chi_{u^{\prime}}\left(u_{i, n} ; r^{\prime}\right)\right)\right| \\
& +C \int_{u_{i, n}}^{u} \frac{\beta_{u^{\prime}}(v) d v}{\left(\chi_{u^{\prime}}\left(v ; r^{\prime}\right)\right)^{4}}+C \int_{u_{i, n}}^{u} \frac{|\Xi(v)|+\Xi^{2}(v)}{\left(\chi_{u^{\prime}}\left(v ; r^{\prime}\right)\right)^{3}} d v .
\end{aligned}
$$

Since $\chi_{u^{\prime}}\left(u ; r^{\prime}\right) \leqq \chi_{u^{\prime}}\left(u_{i, n} ; r^{\prime}\right)$ and $\chi_{u}\left(u_{i, n} ; r^{\prime}\right) \geqq r_{i}$, we have

$$
\left(\chi_{u^{\prime}}\left(u ; r^{\prime}\right)\right)^{2}\left|\frac{\partial h}{\partial u}\left(u_{i, n}, \chi_{u^{\prime}}\left(u_{i, n} ; r^{\prime}\right)\right)\right| \leqq l_{i, n}
$$

where

$$
l_{i, n}:=\sup _{r \geqq r_{r}}\left\{r^{2}\left|\frac{\partial h}{\partial u}\left(u_{i, n}, r\right)\right|\right\} .
$$

Also [see (1.34), (1.36), (1.37)]:

$$
\begin{gathered}
\left(\chi_{u^{\prime}}\left(u ; r^{\prime}\right)\right)^{2} \int_{u_{t, n}}^{u} \frac{\beta_{u^{\prime}}(v)}{\left(\chi_{u^{\prime}}\left(v ; r^{\prime}\right)\right)^{4}} d v \leqq \int_{u_{i, n}}^{u} \frac{\beta_{u^{\prime}}(v)}{\left(\chi_{u^{\prime}}\left(v ; r_{i+1}\right)\right)^{2}} d v \\
\left(\chi_{u^{\prime}}\left(u ; r^{\prime}\right)\right)^{2} \int_{u_{i, n}}^{u} \frac{|\Xi(v)|}{\left(\chi_{u^{\prime}}\left(v ; r^{\prime}\right)\right)^{3}} d v \leqq\left(\int_{u_{l, n}}^{u} \Xi^{2}(v) d v\right)^{1 / 2}\left(\int_{u_{i, n}}^{u} \frac{d v}{\left(\chi_{u^{\prime}}\left(v ; r_{i+1}\right)\right)^{2}}\right)^{1 / 2} \\
\leqq\left(\eta\left(u_{i, n}\right)\right)^{1 / 2}\left(\frac{2}{k_{i} r_{i+1}}\right)^{1 / 2}
\end{gathered}
$$

and

$$
\left(\chi_{u^{\prime}}\left(u ; r^{\prime}\right)\right)^{2} \int_{u_{\imath, n}}^{u} \frac{\Xi^{2}(v)}{\left(\chi_{u^{\prime}}\left(v, r^{\prime}\right)\right)^{3}} d v \leqq \frac{1}{r_{i+1}} \cdot \eta\left(u_{i, n}\right) .
$$

Multiplying then inequality (2.39) by $\left(\chi_{u^{\prime}}\left(u ; r^{\prime}\right)\right)^{2}$ and taking into account (2.40), (2.42), (2.43), and (2.44), we deduce the linear integral inequality

$$
\begin{aligned}
\beta_{u^{\prime}}(u) & =\sup _{r^{\prime} \geqq r_{i+1}}\left\{\left(\chi_{u^{\prime}}\left(u ; r^{\prime}\right)\right)^{2}\left|\frac{\partial h}{\partial u}\left(u, \chi_{u^{\prime}}\left(u ; r^{\prime}\right)\right)\right|\right\} \\
& \leqq l_{i, n}+C\left(\eta^{1 / 2}\left(u_{i, n}\right)+\eta\left(u_{i, n}\right)\right)+C \int_{u_{i, n}}^{u} \frac{\beta_{u^{\prime}}(v)}{\left(\chi_{u^{\prime}}\left(v ; r_{i+1}\right)\right)^{2}} d v,
\end{aligned}
$$


which holds for all $n$ large enough and for all $u^{\prime} \in\left[u_{i+1, n}, u_{n}^{\prime}\right]$ and $u \in\left[u_{i, n}, u^{\prime}\right]$. We conclude that:

$$
\beta_{u^{\prime}}(u) \leqq\left[l_{i, n}+C\left(\eta^{1 / 2}\left(u_{i, n}\right)+\eta\left(u_{i, n}\right)\right)\right] \exp \left[C \int_{u_{i, n}}^{u} \frac{d v}{\left(\chi_{u^{\prime}}\left(v ; r_{i+1}\right)\right)^{2}}\right] .
$$

Therefore, considering that

$$
\int_{u_{i, n}}^{u^{\prime}} \frac{d v}{\left(\chi_{u^{\prime}}\left(v ; r_{i+1}\right)\right)^{2}} \leqq \frac{2}{k_{i} r_{i+1}},
$$

we obtain that for all $u^{\prime} \in\left[u_{i+1, n}, u_{n}^{\prime}\right], n$ large enough,

$$
\sup _{r^{\prime} \geqq r_{\imath+1}}\left\{r^{\prime 2}\left|\frac{\partial h}{\partial u}\left(u^{\prime}, r^{\prime}\right)\right|\right\}=\beta_{u^{\prime}}\left(u^{\prime}\right) \leqq C\left(l_{i, n}+\eta^{1 / 2}\left(u_{i, n}\right)+\eta\left(u_{i, n}\right)\right) .
$$

Now, since $u_{i, n} \rightarrow \infty$ for $n \rightarrow \infty$, we have $\eta\left(u_{i, n}\right) \rightarrow 0$ for $n \rightarrow \infty$. Also, by part 3) of proposition $P_{i}, l_{i, n} \rightarrow 0$ for $n \rightarrow \infty$. It thus follows from (2.46) that

$$
\sup _{A_{\imath}+1, n}\left\{r^{2}\left|\frac{\partial h}{\partial u}\right|\right\} \rightarrow 0 \text { for } n \rightarrow \infty
$$

which together with part 3) of proposition $P_{i}$ implies part 3) of proposition $P_{i+1}$. We conclude that proposition $P_{i}$ implies proposition $P_{i+1}$, and therefore proposition $P_{i}$ is true for each $i$.

It then follows from Lemma 3 [see (2.17)] that for each $i$ and $n \geqq N_{i}$ :

$$
\inf _{A_{i, n}} \bar{g} \geqq \frac{\pi \varepsilon^{2}}{4 M_{1}} \cdot \frac{1}{r_{i}} .
$$

Now since [see (2.14)],

$$
r_{i} \leqq\left(e^{-\pi \varepsilon^{2} / 8 M_{1} r_{0}}\right)^{i} r_{0},
$$

there is a first $l$ such that

$$
r_{l} \leqq \frac{\pi \varepsilon^{2}}{4 M_{1}}
$$

Then according to (2.47) in $A_{l, n}$ for each $n \geqq N_{l}$ we must have $\bar{g} \geqq 1$ : a contradiction. The contradiction is avoided only if $N \rightarrow 0$ as $u \rightarrow \infty$.

Lemma 5. For each $r_{0}>2 M_{1}$,

$$
\sup _{r>r_{0}}\{r|\bar{h}(u, r)|\} \rightarrow 0 \quad \text { as } u \rightarrow \infty \text {, }
$$

and for each $\varepsilon>0$,

$$
\sup _{r \geqq r_{0}}\left\{r^{2-\varepsilon}|h(u, r)|\right\} \rightarrow 0 \text { as } u \rightarrow \infty
$$

Proof. Let us set

$$
\varepsilon\left(u_{0}\right):=\sup _{u \geqq u_{0}}|N(u)| .
$$


According to Lemma $4, \varepsilon\left(u_{0}\right) \rightarrow 0$ as $u_{0} \rightarrow \infty$. Let $\chi_{u_{1}}\left(\cdot ; r_{0}\right)$ denote the characteristic through $\left(u_{1}, r_{0}\right)$, where $r_{0}$ is fixed and greater than $2 M_{1}$. For each $u \in\left[0, u_{1}\right]$, we set

$$
\alpha_{u_{1}}(u):=\sup _{r \geqq \chi_{u_{1}}\left(u ; r_{0}\right)}\left\{r^{3 / 2}|h(u, r)|\right\},
$$

$\alpha_{u_{1}}$ is a continuous function on $\left[0, u_{1}\right]$. By (1.19), for each $u \in\left[0, u_{1}\right]$ and $r \geqq \chi_{u_{1}}\left(u ; r_{0}\right)$, we have

$$
\begin{aligned}
|\bar{h}(u, r)| & \leqq \frac{|N(u)|}{r}+\frac{1}{r} \int_{r}^{\infty}\left|h\left(u, r^{\prime}\right)\right| d r^{\prime} \leqq \frac{|N(u)|}{r}+\frac{1}{r} \int_{r}^{\infty} \alpha_{u_{1}}(u) \frac{d r^{\prime}}{r^{\prime 3 / 2}} \\
& =\frac{|N(u)|}{r}+\frac{2 \alpha_{u_{1}}(u)}{r^{3 / 2}} .
\end{aligned}
$$

In view of (2.50), (2.49), and (1.5), we conclude from the nonlinear evolution equation that in the region $\left\{(u, r) \mid 0 \leqq u<u_{1}, r \geqq \chi_{u_{1}}\left(u ; r_{0}\right)\right\}$,

$$
|D h| \leqq M_{0}\left(\frac{3 \alpha_{u_{1}}}{r^{7 / 2}}+\frac{|N|}{r^{3}}\right) .
$$

holds.

Let $r_{1} \geqq r_{0}$ and let $\chi_{u_{1}}\left(\cdot ; r_{1}\right)$ denote the characteristic through $\left(u_{1}, r_{1}\right)$. Integrating inequality (2.51) along such a characteristic we obtain:

$$
\begin{aligned}
\left|h\left(u, \chi_{u_{1}}\left(u ; r_{1}\right)\right)\right| \leqq & \left|h\left(u_{0}, \chi_{u_{1}}\left(u_{0} ; r_{1}\right)\right)\right| \\
& +3 M_{0} \int_{u_{0}}^{u} \frac{\alpha_{u_{1}}\left(u^{\prime}\right)}{\left(\chi_{u_{1}}\left(u^{\prime} ; r_{1}\right)^{7 / 2}\right.} d u^{\prime}+M_{0} \int_{u_{0}}^{u} \frac{\left|N\left(u^{\prime}\right)\right|}{\left(\chi_{u_{1}}\left(u^{\prime} ; r_{1}\right)\right)^{3}} d u^{\prime} .
\end{aligned}
$$

Now, since $\chi_{u_{1}}\left(u ; r_{1}\right) \leqq \chi_{u_{1}}\left(u_{0} ; r_{1}\right)$, we have

$$
\left(\chi_{u_{1}}\left(u ; r_{1}\right)\right)^{3 / 2}\left|h\left(u_{0}, \chi_{u_{1}}\left(u_{0} ; r_{1}\right)\right)\right| \leqq d\left(u_{1}, u_{0}\right),
$$

where

$$
d\left(u_{1}, u_{0}\right):=\sup _{r \geqq \chi_{u_{1}}\left(u_{0} ; r_{0}\right)}\left\{r^{3 / 2}\left|h\left(u_{0}, r\right)\right|\right\} .
$$

Also,

$$
\left(\chi_{u_{1}}\left(u ; r_{1}\right)\right)^{3 / 2} \int_{u_{0}}^{u} \frac{\alpha_{u_{1}}\left(u^{\prime}\right)}{\left(\chi_{u_{1}}\left(u^{\prime} ; r_{1}\right)\right)^{7 / 2}} d u^{\prime} \leqq \int_{u_{0}}^{u} \frac{\alpha_{u_{1}}\left(u^{\prime}\right)}{\left(\chi_{u_{1}}\left(u^{\prime} ; r_{1}\right)\right)^{2}} d u^{\prime} \leqq \int_{u_{0}}^{u} \frac{\alpha_{u_{1}}\left(u^{\prime}\right)}{\left(\chi_{u_{1}}\left(u^{\prime} ; r_{0}\right)\right)^{2}} d u,
$$

and

$$
\begin{aligned}
& \left(\chi_{u_{1}}\left(u ; r_{1}\right)\right)^{3 / 2} \int_{u_{0}}^{u} \frac{\left|N\left(u^{\prime}\right)\right| d u^{\prime}}{\left(\chi_{u_{1}}\left(u^{\prime} ; r_{1}\right)\right)^{3}} \leqq \int_{u_{0}}^{u} \frac{\left|N\left(u^{\prime}\right)\right| d u^{\prime}}{\left(\chi_{u_{1}}\left(u^{\prime} ; r_{1}\right)\right)^{3 / 2}} \\
& \quad \leqq \int_{u_{0}}^{u} \frac{\left|N\left(u^{\prime}\right)\right| d u}{\left(\chi_{u_{1}}\left(u^{\prime} ; r_{0}\right)\right)^{3 / 2}} \leqq \varepsilon\left(u_{0}\right) \int_{u_{0}}^{u_{1}} \frac{d u^{\prime}}{\left(\chi_{u_{1}}\left(u^{\prime} ; r_{0}\right)\right)^{3 / 2}} \\
& \quad \leqq \frac{2}{k} \varepsilon\left(u_{0}\right) \int_{r_{0}}^{\chi_{u_{1}}\left(u_{0} ; r_{0}\right)} \frac{d r^{\prime}}{r^{\prime 3 / 2}} \leqq \frac{4}{k r_{0}^{1 / 2}} \varepsilon\left(u_{0}\right)
\end{aligned}
$$


[see (1.2)]. Multiplying then (2.52) by $\left(\chi_{u_{1}}\left(u ; r_{1}\right)\right)^{3 / 2}$ and taking into account $(2.53)$ and (2.56), we deduce the linear integral inequality

$$
\begin{aligned}
\alpha_{u_{1}}(u) & =\sup _{r \geqq r_{0}}\left\{\left(\chi_{u_{1}}\left(u ; r_{1}\right)\right)^{3 / 2}\left|h\left(u, \chi_{u_{1}}\left(u ; r_{1}\right)\right)\right|\right\} \\
& \leqq d\left(u_{1}, u_{0}\right)+\frac{4 M_{0}}{k r_{0}^{1 / 2}} \varepsilon\left(u_{0}\right)+3 M_{0} \int_{u_{0}}^{u} \frac{\alpha_{u_{1}}\left(u^{\prime}\right)}{\left(\chi_{u_{1}}\left(u^{\prime} ; r_{0}\right)\right)^{2}} d u^{\prime} .
\end{aligned}
$$

We conclude that

$$
\alpha_{u_{1}}(u) \leqq\left(d\left(u_{1}, u_{0}\right)+\frac{4 M_{0}}{k r_{0}^{1 / 2}} \varepsilon\left(u_{0}\right)\right) \exp \left[3 M_{0} \int_{u_{0}}^{u} \frac{d u^{\prime}}{\left(\chi_{u_{1}}\left(u^{\prime} ; r_{0}\right)\right)^{2}}\right] .
$$

Therefore [sec (1.35)]:

$$
\sup _{r \geqq r_{0}}\left(r^{3 / 2}\left|h\left(u_{1}, r\right)\right|\right\}=\alpha_{u_{1}}\left(u_{1}\right) \leqq e^{6 M_{0} / k r_{0}}\left(d\left(u_{1}, u_{0}\right)+\frac{4 M_{0}}{k r_{0}^{1 / 2}} \varepsilon\left(u_{0}\right)\right) .
$$

According to Lemma 1 , for all $u \geqq 0$ and $r \geqq r_{0}$,

$$
|h(u, r)| \leqq \frac{C}{r^{2}}
$$

holds. This together with (1.41) implies that

$$
d\left(u_{1}, u_{0}\right) \leqq \frac{C}{\left(r_{0}+\frac{k}{2}\left(u_{1}-u_{0}\right)\right)^{1 / 2}}
$$

Given now any $\delta>0$, we first choose $u_{0}$ large enough so that

$$
\varepsilon\left(u_{0}\right) \leqq \delta \cdot \frac{k r_{0}^{1 / 2}}{8 M_{0}} e^{-6 M_{0} / k r_{0}} .
$$

We then choose $u_{2}$ large enough so that

$$
r_{0}+\frac{k}{2}\left(u_{2}-u_{0}\right) \geqq \frac{1}{\delta^{2}} \cdot 4 C^{2} e^{12 M_{0} / k r_{0}} .
$$

Then by (2.58), in view of (2.60), for all $u_{1}>u_{2}$, we have

$$
\sup _{r \geqq r_{0}}\left\{r^{3 / 2}\left|h\left(u_{1}, r\right)\right|\right\}<\delta \text {. }
$$

Therefore,

$$
\sup _{r \geqq r_{0}}\left\{r^{3 / 2}|h(u, r)|\right\} \rightarrow 0 \quad \text { as } \quad u \rightarrow \infty
$$

Since

$$
\bar{h}=\frac{N}{r}-\frac{1}{r} \int_{r}^{\infty} h d r
$$

(2.61) together with the fact that $N \rightarrow 0$ as $u \rightarrow \infty$ implies that

$$
\sup _{r \geqq r_{0}}\{r|\bar{h}(u, r)| \rightarrow 0 \quad \text { as } \quad u \rightarrow \infty .
$$


Finally, the uniform estimate (2.59) together with (2.61) imply (as in the last paragraph of the proof of Lemma 2) that for each $\varepsilon>0$,

$$
\sup _{r \geqq r_{0}}\left\{r^{2-\varepsilon}|h(u, r)|\right\} \rightarrow 0 \quad \text { as } \quad u \rightarrow \infty \text {. }
$$

Lemma 5 implies that at each $r>2 M_{1}$,

$$
M(u)-m(u, r)=2 \pi \int_{r}^{\infty} \frac{\bar{g}}{g}(h-\bar{h})^{2} d r \rightarrow 0 \quad \text { as } \quad u \rightarrow \infty .
$$

The proof of Theorem 1 is therefore complete.

Corollary 1. At each $r \neq 2 M_{1}$,

$$
g \rightarrow g_{1}:=\left\{\begin{array}{lll}
1 & \text { for } & r>2 M_{1} \\
0 & \text { for } & r<2 M_{1}
\end{array} \text { as } \quad u \rightarrow \infty\right.
$$

pointwise, uniformly in each $\left[0, r_{1}\right] \cup\left[r_{2}, \infty\left[, r_{1}<2 M_{1}, r_{2}>2 M_{1}\right.\right.$. Also,

$$
g \rightarrow \bar{g}_{1}:=\left\{\begin{array}{lll}
1-2 M_{1} / r & \text { for } & r>2 M_{1} \\
0 & \text { for } & r \leqq 2 M_{1}
\end{array} \text { as } u \rightarrow \infty,\right.
$$

uniformly in $r$.

Proof. It follows directly from Lemma 5 that at each $r_{2}>2 M_{1}$,

$$
1-g\left(u, r_{2}\right)=1-\exp \left[-4 \pi \int_{r_{2}}^{\infty}(h-\bar{h})^{2} \frac{d r}{r}\right] \rightarrow 0 \text { as } u \rightarrow \infty .
$$

Therefore, in view of the fact that $g$ is a montonically nondecreasing function of $r$ at each $u, g \rightarrow 1$ as $u \rightarrow \infty$ uniformly in $\left[r_{2}, \infty\right.$ [. Now, Lemma 1 implies that there exists a constant $K$ such that for all $u \geqq 0$ and $r \geqq 4 M_{1}$,

$$
1-g(u, r) \leqq \frac{K}{r^{2}}
$$

Therefore in $] 2 M_{1}, \infty[, 1-g(u, \cdot)$ is bounded by a function of $r$ which belongs to $L^{1}\left(2 M_{1}, \infty\right)$ and converges pointwise to 0 for $u \rightarrow \infty$. By the dominated convergence theorem we conclude that

$$
\int_{2 M_{1}}^{\infty}(1-g(u, r)) d r \rightarrow 0 \quad \text { as } \quad u \rightarrow \infty .
$$

Since $\bar{g}$ is expressed as

$$
\bar{g}(u, r)=1-\frac{2 M(u)}{r}+\frac{1}{r} \int_{r}^{\infty}\left(1-g\left(u, r^{\prime}\right)\right) d r^{\prime},
$$

and $M(u) \rightarrow M_{1}$ as $u \rightarrow \infty$, it follows from (2.63) that in the interval [2$M_{1}, \infty[, \bar{g}$ converges uniformly to $1-2 M_{1} / r$ as $u \rightarrow \infty$. In particular, $\bar{g}\left(u, 2 M_{1}\right) \rightarrow 0$ as $u \rightarrow \infty$. Then, in view of the fact that $\bar{g}$ is a monotonically nondecreasing function of $r$ at each $u$, in the interval $\left[0,2 M_{1}\right] \bar{g} \rightarrow 0$ uniformly as $u \rightarrow \infty$. We shall finally show that at each $r_{1}<2 M_{1} g\left(u, r_{1}\right) \rightarrow 0$ as $u \rightarrow \infty$. For if there is an $r_{1}<2 M_{1}$ such that $g(u, r)$ does not tend to 0 as $u \rightarrow \infty$, then there is an $\varepsilon>0$ and a sequence $\left\{u_{n}\right\}, u_{n} \rightarrow \infty$ for $n$ 
$\rightarrow \infty$, such that $g\left(u_{n}, r_{1}\right) \geqq \varepsilon$. But then, since $\bar{g}$ is the mean value function of $g$, we must have $\bar{g}\left(u_{n}, 2 M_{1}\right) \geqq \varepsilon\left(2 M_{1}-r_{1}\right) / 2 M_{1}$, which contradicts the fact that $\bar{g}\left(u, 2 M_{1}\right)$ $\rightarrow 0$ as $u \rightarrow \infty$. Hence $g\left(u, r_{1}\right) \rightarrow 0$ as $u \rightarrow \infty$ for every $r_{1}<2 M_{1}$ and, since $g$ is a monotonically nondecreasing function of $r$ at each $u, g \rightarrow 0$ as $u \rightarrow \infty$ uniformly in $\left[0, r_{1}\right]$.

According to the above corollary, in the region exterior to the Schwarzschild sphere $r=2 M_{1}$ corresponding to the final total mass $M_{1}$, the spacetime metric tends to the Schwarzschild metric

$$
d s^{2}=-\left(1-\frac{2 M_{1}}{r}\right) d u^{2}-2 d u d r+r^{2} d \Sigma^{2},
$$

as the retarded time $u$ tends to infinity.

\section{The Formation of the Event Horizon}

In Proposition 1 of [1] it was shown that the part of the limiting hypersurface $u=\infty$ for which $r>2 M_{1}$ represents future timelike infinity. In the present section it will be shown that the part of the limiting hypersurface $u=\infty$ for which $r<2 M_{1}$ represents the future event horizon. This will be so as we shall demonstrate that the timelike lines $r=r_{1}$ for $r_{1}<2 M_{1}$ are incomplete, that is, they have finite proper length. To prove this we shall estimate the rate at which $g\left(u, r_{1}\right)$ tends to 0 as $u \rightarrow \infty$. The results of this section are contained in:

Theorem 2. In the interval $\left[0,2 M_{1}\right.$ [ there is a continuous increasing function $u_{0}(r)$ such that in the region

$$
\left\{(u, r) \mid u \geqq u_{0}(r), \quad r \in\left[0,2 M_{1}[\},\right.\right.
$$

we have

$$
g(u, r) \leqq e^{-\left(u-u_{0}(r)\right) / 32 M_{1}} .
$$

For each $r_{1} \in\left[0,2 M_{1}\right.$ [ the timelike lines $r=r_{1}$ are incomplete, and their proper length $T\left(r_{1}\right)$ is a continuous increasing function in $\left[0,2 M_{1}[\right.$. Also for each $\left.r_{1} \in\right] 0,2 M_{1}\left[\right.$ there is a unique characteristic $\chi_{r_{1}}$ asymptotic to the line $r=r_{1}$ as $u \rightarrow \infty$.

Proof. For any generalized solution we can show that the quantity

$$
\int_{0}^{\infty} r h^{2} d r
$$

is a continously differentiable function of $u$ and

$$
4 \pi \frac{\partial}{\partial u}\left(\int_{0}^{\infty} r h^{2} d r\right)=M-\int_{0}^{\infty} g \log (1 / g) d r .
$$

We call (3.1) the "scaling identity" as it arises from the covariance of the nonlinear evolution equation under the scaling group $(u, r) \rightarrow(u / a, r / a), a>0$ (see Sect. 4 of [1]). In fact, the nonlinear evolution equation arises from the variational principle 
corresponding to the action:

$$
S(g, \bar{g}, \bar{h} ; u)=\int_{0}^{u} d u \int_{0}^{\infty} d r\left\{\frac{1}{4 \pi}\left(1-g-\frac{r}{g} \frac{\partial g}{\partial r} \bar{g}\right)+r^{2}\left[\bar{g}\left(\frac{\partial \bar{h}}{\partial r}\right)^{2}-2 \frac{\partial \bar{h}}{\partial u}\left(\frac{\partial \bar{h}}{\partial r}+\frac{\bar{h}}{r}\right)\right]\right\},
$$

where the quantities $g, \bar{g}$, and $\bar{h}$ are to be varied independently. Variation with respect to $\bar{g}$ gives the definition of $g$, variation with respect to $g$ gives the definition of $\bar{g}$ and variation with respect to $\bar{h}$ gives the nonlinear evolution equation. The action (3.2) is covariant under the scaling group in the sense that $g^{\prime}(u, r)$ $=g(u / a, r / a), \quad \bar{g}^{\prime}(u, r)=\bar{g}(u / a, r / a), \quad \overline{h^{\prime}}(u, r)=\bar{h}(u / a, r / a), \quad$ then $\quad S\left(g^{\prime}, \bar{g}^{\prime}, \bar{h}^{\prime} ; u\right)$ $=a^{2} S(g, \bar{g}, \bar{h} ; u / a)$. The identity (3.1) arises through Noether's theorem from this covariance as the identity

$$
\frac{\partial M}{\partial u}=-\pi \Xi^{2}
$$

arises from the invariance of $S$ under the group of time translations $u \rightarrow u+b$.

By Corollary 1, at each $r \neq 2 M_{1}, g \log (1 / g) \rightarrow g_{1} \log \left(1 / g_{1}\right)$ as $u \rightarrow \infty$. But $g_{1} \log \left(1 / g_{1}\right)=0$, since if $f(x):=x \log (1 / x)$, then $f(0)=f(1)=0$. We have $g \log (1 / g) \leqq e^{-1}$, since the maximum value of $f$ in the interval $[0,1]$ is equal to $e^{-1}$. Also, (2.62) implies that there is a constant $K^{\prime}$ such that at all $u$ and $r \in\left[4 M_{1}, \infty[\right.$ :

$$
g \log (1 / g) \leqq K^{\prime} / r^{2}
$$

Thus $g(u, \cdot) \log (1 / g(u, \cdot))$ is dominated as an integrable function of $r$ and converges to 0 pointwise for almost all $r$ as $u \rightarrow \infty$. By the dominated convergence theorem we conclude that

$$
\int_{0}^{\infty} g \log (1 / g) d r \rightarrow 0 \quad \text { as } \quad u \rightarrow \infty
$$

Therefore there is a $u_{1}$ such that for all $u \geqq u_{1}$,

$$
\int_{0}^{\infty}(g \log (1 / g))(u, r) d r \leqq \frac{M_{1}}{2} .
$$

The scaling identity (3.3) then implies that if $u \geqq u_{1}$,

$$
\int_{0}^{\infty} r h^{2}(u, r) d r \geqq \frac{M_{1}}{8 \pi}\left(u-u_{1}\right) .
$$

The proof of Theorem 2 is based on the local form of the scaling identity, that is, the evolution law of the function

$$
\int_{r}^{\infty} r h^{2} d r
$$

along the characteristics. We have

$$
\begin{aligned}
D\left(\int_{r}^{\infty} r h^{2} d r\right)=\int_{r}^{\infty} \frac{\partial}{\partial u}\left(r h^{2}\right)+\frac{1}{2} \bar{g} r h^{2} & =\int_{r}^{\infty}\left[D\left(r h^{2}\right)+\frac{1}{2} \bar{g} \frac{\partial}{\partial r}\left(r h^{2}\right)\right] d r+\frac{1}{2} \bar{g} r h^{2} \\
& =\int_{r}^{\infty}\left[D\left(r h^{2}\right)-\frac{1}{2}\left(\frac{\partial \bar{g}}{\partial r}\right) r h^{2}\right] d r .
\end{aligned}
$$


From the nonlinear evolution equation we obtain

$$
D\left(r h^{2}\right)-\frac{1}{2}\left(\frac{\partial \bar{g}}{\partial r}\right) r h^{2}=-\frac{1}{2} \bar{g} h^{2}+\frac{1}{2}(g-\bar{g})(h-\bar{h})^{2}-\frac{1}{2}(g-\bar{g}) \bar{h}^{2} .
$$

Now, since $\partial\left(r \bar{h}^{2}\right) / \partial r=2 h \bar{h}-\bar{h}^{2}$, we have:

$$
\int_{r}^{\infty} \bar{g} h^{2} d r=\int_{r}^{\infty} \bar{g}(h-\bar{h})^{2} d r+\int_{r}^{\infty} \bar{g} \frac{\partial}{\partial r}\left(r \bar{h}^{2}\right) d r=\int_{r}^{\infty} \bar{g}(h-\bar{h})^{2} d r-r \bar{g} \bar{h}^{2}-\int_{r}^{\infty}(g-\bar{g}) \bar{h}^{2} d r .
$$

We therefore obtain from (3.6):

$$
\int_{r}^{\infty}\left[D\left(r h^{2}\right)-\frac{1}{2}\left(\frac{\partial \bar{g}}{\partial r}\right) r h^{2}\right] d r=\frac{1}{2} r \bar{g} \bar{h}^{2}+\frac{1}{2} \int_{r}^{\infty} g(h-\bar{h})^{2} d r-\int_{r}^{\infty} \bar{g}(h-\bar{h})^{2} d r .
$$

We can express:

$$
\int_{r}^{\infty} g(h-\bar{h})^{2} d r=-\frac{1}{4 \pi} \int_{r}^{\infty} r \frac{\partial}{\partial r}(1-g) d r=\frac{r}{4 \pi}(1-g)+\frac{1}{4 \pi} \int_{r}^{\infty}(1-g) d r,
$$

and

$$
\int_{r}^{\infty} \bar{g}(h-\bar{h})^{2} d r=\frac{1}{4 \pi} \int_{r}^{\infty} r \bar{g} \frac{\partial \log g}{\partial r} d r=\frac{r}{4 \pi} \bar{g} \log (1 / g)+\frac{1}{4 \pi} \int_{r}^{\infty} g \log (1 / g) d r .
$$

In view of (3.5), (3.7), (3.8), and (3.9) we conclude that:

$$
\begin{aligned}
D\left(\int_{r}^{\infty} r h^{2} d r\right)= & \frac{1}{2} r \bar{g} \bar{h}^{2}+\frac{r}{8 \pi}(1-g)+\frac{1}{8 \pi} \int_{r}^{\infty}(1-g) d r \\
& -\frac{r}{4 \pi} \bar{g} \log (1 / g)-\frac{1}{4 \pi} \int_{r}^{\infty} g \log (1 / g) d r .
\end{aligned}
$$

This is what we call the "local scaling identity."

We shall now show that the local scaling identity together with Corollary 1 imply that in the interval $\left[0,2 M_{1}\left[\right.\right.$, there is a continuous increasing function $u_{1}(r)$ such that in the region

$$
\left\{(u, r) \mid u \geqq u_{1}(r), \quad r \in\left[0,2 M_{1}[\},\right.\right.
$$

we have

$$
D\left(\int_{r}^{\infty} r h^{2} d r\right) \geqq \frac{M_{1}}{8 \pi} .
$$

Indeed, since, according to Corollary $1, g(u, \cdot)$ tends to 0 as $u \rightarrow \infty$ pointwise in $\left[0,2 M_{1}\right.$ [ and $g$ is a continuous increasing function of $r$ at each $u$, we can find in $\left[0,2 M_{1}\left[\right.\right.$ a continuous increasing function $u_{2}(r)$ such that for each $r \in\left[0,2 M_{1}[\right.$, $u \geqq u_{2}(r)$ implies

$$
g(u, r) \leqq 1 / 6
$$

and

$$
(g \log (1 / g))(u, r) \leqq 1 / 12
$$


Also, by (3.3) there is a positive real number $u_{3}$ such that $u \geqq u_{3}$ implies

$$
\int_{0}^{\infty}(g \log (1 / g))(u, r) d r \leqq \frac{M_{1}}{6} .
$$

We set

$$
u_{1}(r):=\max \left\{u_{2}(r), u_{3}\right\}
$$

Then, considering the fact that

$$
r(1-g)+\int_{r}^{\infty}(1-g) d r>2 M-r g, \quad\left(M=\frac{1}{2} \int_{0}^{\infty}(1-g) d r\right),
$$

(3.10) implies that at each $(u, r)$ such that $0 \leqq r<2 M_{1}$ and $u \geqq u_{1}(r)$, (3.12) holds.

We shall now show that there is a constant $\gamma$ such that for all $u \geqq 0$ and $r \leqq 4 M_{1}$, we have

$$
g(u, r) \leqq \exp \left[\gamma-\frac{\pi}{4 M_{1}^{2}} \int_{r}^{\infty} r h^{2} d r\right]
$$

Indeed, since

$$
\int_{r_{1}}^{4 M_{1}}(h-\bar{h})^{2} d r=\int_{r_{1}}^{4 M_{1}} h^{2} d r-\int_{r_{1}}^{4 M_{1}} \frac{\partial}{\partial r}\left(r \overline{h^{2}}\right) d r=\int_{r_{1}}^{4 M_{1}} h^{2} d r-4 M_{1} \overline{h^{2}}\left(4 M_{1}\right)+r_{1} \bar{h}^{2}\left(r_{1}\right),
$$

we have by Lemma 1:

$$
\int_{r_{1}}^{4 M_{1}}(h-\bar{h})^{2} d r \geqq \int_{r_{1}}^{4 M_{1}} h^{2} d r-\frac{B^{2}}{4 M_{1}} .
$$

Therefore:

$$
\begin{aligned}
\int_{r_{1}}^{4 M_{1}}(h-\bar{h})^{2} \frac{d r}{r} & \geqq \frac{1}{4 M_{1}} \int_{r_{1}}^{4 M_{1}}(h-\bar{h})^{2} d r \geqq \frac{1}{4 M_{1}} \int_{r_{1}}^{4 M_{1}} h^{2} d r-\frac{B^{2}}{16 M_{1}^{2}} \\
& \geqq \frac{1}{16 M_{1}^{2}}\left(\int_{r_{1}}^{4 M_{1}} r h^{2} d r-B^{2}\right) .
\end{aligned}
$$

On the other hand, again by Lemma 1,

$$
\int_{r_{1}}^{4 M_{1}} r h^{2} d r=\int_{r_{1}}^{\infty} r h^{2} d r-\int_{4 M_{1}}^{\infty} r h^{2} d r \geqq \int_{r_{1}}^{\infty} r h^{2} d r-\int_{4 M_{1}}^{\infty} \frac{C^{2}}{r^{3}} d r=\int_{r_{1}}^{\infty} r h^{2} d r-\frac{C^{2}}{32 M_{1}^{2}} .
$$

Consequently, defining

$$
\gamma:=\frac{\pi}{4 M_{1}^{2}}\left(B^{2}+\frac{C^{2}}{32 M_{1}^{2}}\right),
$$

we obtain from (3.14) and (3.15) that

$$
A\left(r_{1}\right):=\int_{r_{1}}^{\infty}(h-\bar{h})^{2} \frac{d r}{r} \geqq \frac{1}{16 M_{1}^{2}} \int_{r_{1}}^{\infty} r h^{2} d r-\frac{\gamma}{4 \pi} .
$$


Then, since $g=e^{-4 \pi A}$, inequality (3.13) follows.

Given now $\left.r_{1} \in\right] 0,2 M_{1}\left[\right.$, let $r_{0}$ be the arithmetic mean of $r_{1}$ and $2 M_{1}$ :

$$
r_{0}:=\frac{1}{2}\left(r_{1}+2 M_{1}\right) \text {, }
$$

and let $\left\{u_{n}^{\prime \prime} \mid n=1,2, \ldots\right\}$ be an increasing sequence of positive real numbers such that $u_{n}^{\prime \prime} \rightarrow \infty$ for $n \rightarrow \infty$. Consider the sequence of points $\left(u_{n}^{\prime \prime}, r_{1}\right)$ on the line $r=r_{1}$. Let $u_{n}^{\prime}$ be the value of $u$ at which the characteristic through $\left(u_{n}^{\prime \prime}, r_{1}\right)$ intersects the line $r=r_{0}$. Then the sequence $\left\{u_{n}^{\prime} \mid n=1,2, \ldots\right\}$ is increasing. For each $n$, let us denote by $\chi_{n}$ the segment of the characteristic through $\left(u_{n}^{\prime \prime}, r_{1}\right)$ between that point and the point $\left(u_{n}^{\prime}, r_{0}\right)$. We shall show that the sequence $\left\{u_{n}^{\prime}\right\}$ has an upper bound. For, either

Case 1) $u_{n}^{\prime}<u_{1}\left(r_{0}\right)$ for all $n$,

or,

Case 2) $u_{n}^{\prime} \geqq u_{1}\left(r_{0}\right)$ from some $n$ onward,

In case 1 ) the sequence $\left\{u_{n}^{\prime}\right\}$ has obviously an upper bound. In case 2 ) for all large enough $n$ the segment $\chi_{n}$ is contained in the region defined by (3.11). Therefore (3.12) holds along $\chi_{n}$ which implies that, along $\chi_{n}$,

$$
\left[\int_{r}^{\infty} r h^{2} d r\right]_{\chi_{n}}(u) \geqq \frac{M_{1}}{8 \pi}\left(u-u_{n}^{\prime}\right) .
$$

This in turn implies by (3.13) that along $\chi_{n}$ :

$$
[g]_{\chi_{n}}(u) \leqq e^{\gamma} \cdot e^{-\left(u-u_{n}^{\prime}\right) / 32 M_{1}},
$$

and, a fortiori,

$$
[\bar{g}]_{\chi_{n}}(u) \leqq e^{\gamma} \cdot e^{-\left(u-u_{n}^{\prime}\right) / 32 M_{1}} .
$$

Let us set

$$
\theta(u):=\sup _{u^{\prime} \in[u, \infty,[} \bar{g}\left(u^{\prime}, 2 M_{1}\right) .
$$

Then $\theta$ is a continuous decreasing function of $u$ and, according to Corollary $1, \theta(u)$ $\rightarrow 0$ for $u \rightarrow \infty$. Since $[\bar{g}]_{\chi_{n}}(u) \leqq \bar{g}\left(u, 2 M_{1}\right)$, we have:

$$
[g]_{\chi_{n}}(u) \leqq \theta\left(u_{n}^{\prime}\right)
$$

We conclude that along $\chi_{n}, \bar{g}$ is bounded by the geometric mean of the right-hand sides of (3.21) and (3.19):

$$
[\bar{g}]_{\chi_{n}}(u) \leqq e^{\gamma / 2}\left(\theta\left(u_{n}^{\prime}\right)\right)^{1 / 2} e^{-\left(u-u_{n}^{\prime}\right) / 64 M_{1}} .
$$

According to the definition of $u_{n}^{\prime}$, we have

$$
r_{0}-r_{1}=\frac{1}{2} \int_{u_{n}^{\prime}}^{u_{n}^{\prime \prime}}[\bar{g}]_{\chi_{n}}(u) d u \text {. }
$$

Therefore, by (3.22):

$$
\frac{2 M_{1}-r_{1}}{2}=r_{0}-r_{1} \leqq \frac{1}{2} e^{\gamma / 2}\left(\theta\left(u_{n}^{\prime}\right)\right)^{1 / 2} \int_{u_{n}^{\prime}}^{\infty} e^{-\left(u-u_{n}^{\prime}\right) / 64 M_{1}} d u=32 M_{1} e^{\gamma / 2}\left(\theta\left(u_{n}^{\prime}\right)\right)^{1 / 2} .
$$


Hence

$$
\theta\left(u_{n}^{\prime}\right) \geqq \frac{e^{-\gamma}}{\left(32 M_{1}\right)^{2}}\left[\frac{2 M_{1}-r_{1}}{2}\right]^{2} .
$$

Since $\theta(u) \rightarrow 0$ for $u \rightarrow \infty,(3.23)$ implies that for each $\left.r_{1} \in\right] 0,2 M_{1}\left[\right.$ the sequence $\left\{u_{n}^{\prime}\right\}$ has, in case 2$)$, an upper bound $b\left(r_{1}\right)$ which increases with $r_{1} . b$ may be chosen to depend continously on $r_{1}$.

We conclude from the above that for each $\left.r_{1} \in\right] 0,2 M_{1}[$,

$$
u_{n}^{\prime} \rightarrow u^{\prime}\left(r_{1}\right):=\sup _{n}\left\{u_{n}^{\prime}\right\} \text { as } n \rightarrow \infty,
$$

and

$$
u^{\prime}\left(r_{1}\right) \leqq u_{0}^{\prime}\left(r_{1}\right):=\max \left\{u_{1}\left(r_{0}\right), b\left(r_{1}\right)\right\} .
$$

Then the characteristic through $\left(u^{\prime}\left(r_{1}\right), r_{0}\right)$, extended into the future, is asymptotic to the line $r=r_{1}$ as $u \rightarrow \infty$. Let $\chi_{r_{1}}$ denote this characteristic. Then the part of $\chi_{r_{1}}$ to the future of the line $u=u_{0}^{\prime}\left(r_{1}\right)$ lies in the region (3.11). Therefore for $u \geqq u_{0}^{\prime}\left(r_{1}\right),(3.12)$ holds along $\chi_{r_{1}}$, which implies that:

$$
\left[\int_{r}^{\infty} r h^{2} d r\right]_{\chi_{r_{1}}}(u) \geqq \frac{M_{1}}{8 \pi}\left(u-u_{0}^{\prime}\left(r_{1}\right)\right) .
$$

This in turn implies by (3.13):

$$
[g]_{\chi_{r_{1}}}(u) \leqq e^{\gamma} \cdot e^{-\left(u-u_{0}^{\prime}\left(r_{1}\right)\right) / 32 M_{1}} .
$$

Consequently, setting

$$
u_{0}\left(r_{1}\right):=u_{0}^{\prime}\left(r_{1}\right)+32 M_{1} \gamma
$$

$u_{0}\left(r_{1}\right)$ is a continuous increasing function of $r_{1} \in\left[0,2 M_{1}\right.$ [, and along $\chi_{r_{1}}$ for $u \geqq u_{0}\left(r_{1}\right)$,

$$
[g]_{\chi_{r_{1}}}(u) \leqq e^{-\left(u-u_{0}\left(r_{1}\right)\right) / 32 M_{1}} .
$$

holds. Therefore, a fortiori,

$$
g\left(u, r_{1}\right) \leqq e^{-\left(u-u_{0}\left(r_{1}\right)\right) / 32 M_{1}}
$$

holds for all $r_{1} \in\left[0,2 M_{1}\left[\right.\right.$ and all $u \geqq u_{0}\left(r_{1}\right)$.

The proper time element along the line $r=r_{1}$ is $e^{v\left(u, r_{1}\right)} d u$, and $e^{v}=(g \bar{g})^{1 / 2}$ (see part I). By (3.27), $(g \bar{g})^{1 / 2}\left(\cdot, r_{1}\right) \in L^{1}(0, \infty)$ for each $r_{1} \in\left[0,2 M_{1}[\right.$. Therefore the proper length of the lines $r=r_{1}$,

$$
T\left(r_{1}\right)=\int_{0}^{\infty}(g \bar{g})^{1 / 2}\left(u, r_{1}\right) d u \leqq u_{0}\left(r_{1}\right)+32 M_{1}
$$

is finite for each $r_{1} \in\left[0,2 M_{1}\left[\right.\right.$. The fact that the function $(g \bar{g})^{1 / 2}$ is continuous and monotone with respect to $r$ implies by the monotone convergence theorem that if $\left\{r_{n}\right\}$ is an increasing or decreasing sequence in $\left[0,2 M_{1}\left[\right.\right.$ such that $r_{n} \rightarrow r \in\left[0,2 M_{1}[\right.$, then

$$
T\left(r_{n}\right) \rightarrow T(r)
$$


Therefore the function $T$ is continuous in $\left[0,2 M_{1}[\right.$.

We shall finally demonstrate that for each $\left.r_{1} \in\right] 0,2 M_{1}\left[\right.$ the characteristic $\chi_{r_{1}}$ constructed above is the only characteristic asymptotic to the line $r=r_{1}$ as $u \rightarrow \infty$. For, let $\chi^{\prime}$ and $\chi^{\prime \prime}$ be two characteristics which are both asymptotic to the line $r=r_{1}$ as $u \rightarrow \infty$. Their equations are $r=\chi^{\prime}(u)$ and $r=\chi^{\prime \prime}(u)$ respectively, and $\chi^{\prime}(u) \rightarrow r_{1}, \chi^{\prime \prime}$ $\rightarrow r_{1}$ for $u \rightarrow \infty$. We can assume that $\chi^{\prime}(u)<\chi^{\prime \prime}(u)$ at some, and therefore all, $u$. Then if $r_{0}:=\left(r_{1}+2 M_{1}\right) / 2$, there exists a $u_{1}$ such that $\chi^{\prime \prime}\left(u_{1}\right)=r_{0}$. Consider the characteristics through any point $r=s$ on the line $u=u_{1}$ such that $\chi^{\prime}\left(u_{1}\right)<s<\chi^{\prime \prime}\left(u_{1}\right)$. Such a characteristic, the equation of which we denote by $r=\chi_{u_{1}}(u ; s)$, must also be asymptotic to the line $r=r_{1}$ as $u \rightarrow \infty$. According to Sect. 5 of [1] (the convergence factor) for any $u_{2}>u_{1}$ we have:

$$
\begin{aligned}
\chi^{\prime \prime}\left(u_{2}\right)-\chi^{\prime}\left(u_{2}\right)= & \left(\chi^{\prime \prime}\left(u_{1}\right)-\chi^{\prime}\left(u_{1}\right)\right) \\
& \times \operatorname{mean}_{s \in\left[\chi^{\prime}\left(u_{1}\right), \chi^{\prime \prime}\left(u_{1}\right)\right]}\left\{\exp \left[-\frac{1}{2} \int_{u_{1}}^{u_{2}}\left[\frac{1}{r}(g-\bar{g})\right]\left(u, \chi_{u_{1}}(u ; s)\right) d u\right]\right\} .
\end{aligned}
$$

Taking into account (3.27) we obtain that for each $s \in\left[\chi^{\prime}\left(u_{1}\right), \chi^{\prime \prime}\left(u_{1}\right)\right]$ :

$$
\int_{u_{1}}^{u_{2}}\left[\frac{1}{r}(g-\bar{g})\right]\left(u, \chi_{u_{1}}(u ; s) d u \leqq \frac{1}{r_{1}} \int_{0}^{\infty} g\left(u, r_{0}\right) d u \leqq \frac{1}{r_{1}}\left(u_{0}\left(r_{0}\right)+32 M_{1}\right)\right. \text {. }
$$

Therefore, by (3.29)

$$
\chi^{\prime \prime}\left(u_{1}\right)-\chi^{\prime}\left(u_{1}\right) \leqq e^{\left(u_{0}\left(r_{0}\right)+32 M_{1}\right) / 2 r_{1}}\left(\chi^{\prime \prime}\left(u_{2}\right)-\chi^{\prime}\left(u_{2}\right)\right) .
$$

Letting $u_{2} \rightarrow \infty$ in (3.31), we obtain $\chi^{\prime \prime}\left(u_{1}\right)-\chi^{\prime}\left(u_{1}\right)=0$. Therefore, $\chi^{\prime \prime}(u)=\chi^{\prime}(u)$ for all $u$.

The proof of Theorem 2 is now complete.

We note that as $r_{1} \rightarrow 2 M_{1}, u_{0}\left(r_{1}\right)$ and $T\left(r_{1}\right)$ tend to infinity. The point $r=2 M_{1}$ on the ideal line $u=\infty$ represents the point at infinity on the future event horizon.

\section{The Behaviour of the Scalar Field on the Horizon}

The following theorem describes the behaviour of the scalar field on the future event horizon:

Theorem 3. At each $r \in] 0,2 M_{1}[$,

$$
\bar{h} \rightarrow \bar{h}_{1}, h \rightarrow h_{1}, \partial h / \partial r \rightarrow \partial h_{1} / \partial r, \quad \text { as } u \rightarrow \infty
$$

pointwise, uniformly in each compact subinterval of the interval $] 0,2 M_{1}\left[. h_{1}\right.$ is a continuously differentiable function on the interval $] 0,2 M_{1}\left[\right.$ and $h_{1} \in L^{2}\left(0, r_{1}\right)$ for each $r_{1}<2 M_{1}$. Also, $\bar{h}_{1}$ is the mean value function of $h_{1}$.

Proof. For each $\left.r_{1} \in\right] 0,2 M_{1}[$, consider the mass-flux relation (Eq. 5.43 of [2]) along the characteristic $\chi_{r_{1}}$ asymptotic to the line $r=r_{1}$ :

$$
m\left(u_{1}, \chi_{r_{1}}\left(u_{1}\right)\right)+\pi \int_{0}^{u_{1}}\left[\frac{1}{g} \xi^{2}\right]_{\nu_{1}}(u) d u=m\left(0, \chi_{r_{1}}(0)\right) .
$$


Since $m$ is nonnegative and a monotonically nonincreasing function of $u$ along $\chi_{r_{1}}$, $m\left(u, \chi_{r_{1}}(u)\right)$ tends to a limit $m_{1}\left(r_{1}\right)$ as $u \rightarrow \infty$,

$$
\lim _{u \rightarrow \infty} m\left(u, \chi_{r_{1}}(u)\right):=m_{1}\left(r_{1}\right) .
$$

Then, letting $u_{1} \rightarrow \infty$ in (4.1), we obtain that

$$
\left[\frac{1}{g^{1 / 2}} \xi\right]_{{\chi r_{1}}_{1}} \in L^{2}(0, \infty)
$$

and

$$
\int_{0}^{\infty}\left[\frac{1}{g^{1 / 2}} \xi^{2}\right]_{\chi_{r_{1}}}(u) d u=\frac{1}{\pi}\left(m\left(0, \chi_{r_{1}}(0)\right)-m_{1}\left(r_{1}\right)\right) .
$$

Integrating the evolution law of $\bar{h}(D \bar{h}=\xi / 2 r)$ along $\chi_{r_{1}}$, we obtain

$$
\bar{h}\left(u_{2}, \chi_{r_{1}}\left(u_{2}\right)\right)-\bar{h}\left(u_{1}, \chi_{r_{1}}\left(u_{1}\right)\right)=\int_{u_{1}}^{u_{2}}\left[\frac{\xi}{2 r}\right]_{\chi_{r_{1}}}(u) d u .
$$

Therefore

$$
\left|\bar{h}\left(u_{2}, \chi_{r_{1}}\left(u_{2}\right)\right)-\bar{h}\left(u_{1}, \chi_{r_{1}}(u)\right)\right| \leqq \frac{1}{2 r_{1}}\left(\int_{u_{1}}^{u_{2}}\left[\frac{\xi^{2}}{g}\right]_{\chi_{r_{1}}}(u) d u\right)^{1 / 2}\left(\int_{u_{1}}^{u_{2}}[g]_{\chi_{r_{1}}}(u) d u\right)^{1 / 2} .
$$

Now, by (4.3),

$$
\int_{u_{1}}^{u_{2}}\left[\frac{\xi^{2}}{g}\right]_{\chi_{r_{1}}}(u) d u \leqq \frac{M_{0}}{\pi},
$$

while by (3.26) for $u_{1} \geqq u_{0}\left(r_{1}\right)$, we have

$$
\int_{u_{1}}^{u_{2}}[g]_{\chi_{r_{1}}} d u \leqq 32 M_{1} e^{u_{0}\left(r_{1}\right) / 32 M_{1}}\left(e^{-u_{1} / 32 M_{1}}-e^{-u_{2} / 32 M_{1}}\right) .
$$

Hence

$$
\begin{aligned}
\left|\bar{h}\left(u_{2}, \chi_{r_{1}}\left(u_{2}\right)\right)-\bar{h}\left(u_{1}, \chi_{r_{1}}\left(u_{1}\right)\right)\right| \leqq & \frac{16 M_{0} M_{1}}{\pi r_{1}} e^{u_{0}\left(r_{1}\right) / 32 M_{1}}\left(e^{-u_{1} / 32 M_{1}}-e^{-u_{2} / 32 M_{1}}\right) \\
& \rightarrow 0 \text { for } u_{2}>u_{1}, u_{1} \rightarrow \infty .
\end{aligned}
$$

We conclude that $\bar{h}\left(u, \chi_{r_{1}}(u)\right)$ tends to a limit $\bar{h}_{1}\left(r_{1}\right)$ as $u \rightarrow \infty$. Letting $r_{1}$ range over a compact subinterval $[a, b] \subset] 0,2 M_{1}[$ and taking into account the fact that $u_{0}\left(r_{1}\right)$ is an increasing function of $r_{1}$, we obtain

$$
\left|\bar{h}\left(u_{2}, \chi_{r_{1}}\left(u_{2}\right)\right)-\bar{h}\left(u_{1}, \chi_{r_{1}}\left(u_{1}\right)\right)\right| \leqq \frac{16 M_{0} M_{1}}{\pi a} e^{u_{0}(b) / 32 M_{1}}\left(e^{-u_{1} / 32 M_{1}}-e^{-u_{2} / 32 M_{1}}\right) .
$$

We conclude that the convergence is uniform in any compact subinterval of ]0, $2 M_{1}\left[\right.$, and therefore $\bar{h}_{1}$ is a continuous function on $] 0,2 M_{1}[$. that

By the above on the interval $] 0,2 M_{1}\left[\right.$ there is a continuous function $c_{0}$ such

$$
\left|\bar{h}\left(u, \chi_{r_{1}}\left(u_{1}\right)\right)\right| \leqq c_{0}\left(r_{1}\right)
$$


for all $u \geqq 0$ and $\left.r_{1} \in\right] 0,2 M_{1}[$. We now integrate the nonlinear evolution equation along $\chi_{r_{1}}$, obtaining

$$
\begin{aligned}
h\left(u_{1}, \chi_{r_{1}}\left(u_{1}\right)\right)= & \exp \left[\int_{0}^{u_{1}}\left[\frac{1}{2 r}(g-\bar{g})\right]_{\chi_{r_{1}}}(u) d u\right]\left\{h\left(0, \chi_{r_{1}}(0)\right)\right. \\
& \left.-\int_{0}^{u_{1}}\left[\frac{1}{2 r}(g-\bar{g}) \bar{h}\right]_{\gamma_{r_{1}}}(u) \exp \left[-\int_{0}^{u}\left[\frac{1}{2 r}(g-\bar{g})\right]_{\chi_{r_{1}}}\left(u^{\prime}\right) d u^{\prime}\right] d u\right\} .
\end{aligned}
$$

By (3.26) we have:

$$
\int_{0}^{u_{1}}\left[\frac{1}{2 r}(g-\bar{g})\right]_{\chi_{r_{1}}}(u) d u \leqq \frac{1}{2 r_{1}} \int_{0}^{u_{1}}[g]_{\chi_{r_{1}}}(u) d u \leqq \frac{1}{2 r_{1}}\left(u_{0}\left(r_{1}\right)+32 M_{1}\right) .
$$

Taking into account (4.8) and (4.7) we conclude that for all $u_{1} \geqq 0$ and $\left.r_{1} \in\right] 0,2 M_{1}[$,

$$
\left|h\left(u_{1}, \chi_{r_{1}}\left(u_{1}\right)\right)\right| \leqq c_{1}\left(r_{1}\right),
$$

where,

$$
c_{1}\left(r_{1}\right):=e^{\left(u_{0}\left(r_{1}\right)+32 M_{1}\right) / 2 r_{1}}\left[\left|h\left(0, \chi_{r_{1}}(0)\right)\right|+\frac{c_{0}\left(r_{1}\right)}{2 r_{1}}\left(u_{0}\left(r_{1}\right)+32 M_{1}\right)\right] .
$$

$c_{1}$ is a continuous function on the interval $] 0,2 M_{1}\left[\right.$. Let us now take $u_{2}>u_{1}$ $\geqq u_{0}\left(r_{1}\right)$. By (4.9), (4.7), and (4.5), we then have

$$
\begin{aligned}
\mid h\left(u_{2}, \chi_{r_{1}}\left(u_{2}\right)-h\left(u_{1}, \chi_{r_{1}}\left(u_{1}\right)\right) \mid\right. & \leqq \int_{u_{1}}^{u_{2}}[|D h|]_{\chi_{r_{1}}}(u) d u=\int_{u_{1}}^{u_{2}}\left[\frac{1}{2 r}(g-\bar{g})|h-\bar{h}|\right]_{\chi_{r_{1}}}(u) d u \\
& \leqq k\left(r_{1}\right)\left(e^{-u_{1} / 32 M_{1}}-e^{-u_{2} / 32 M_{1}}\right)
\end{aligned}
$$

where

$$
k\left(r_{1}\right):=\frac{16 M_{1}}{r_{1}}\left(c_{0}\left(r_{1}\right)+c_{1}\left(r_{1}\right)\right) e^{u_{0}\left(r_{1}\right) / 32 M_{1}} .
$$

Since $k$ is a continuous function on $] 0,2 M_{1}[,(4.11)$ implies that in any compact subinterval $[a, b]$ of the interval $] 0,2 M_{1}\left[,\left|h\left(u_{2}, \chi_{r_{1}}\left(u_{2}\right)\right)-h\left(u_{1}, \chi_{r_{1}}\left(u_{1}\right)\right)\right| \rightarrow 0\right.$ as, $u_{2}>u_{1}, u_{1} \rightarrow \infty$, uniformly in $r_{1} \in[a, b]$. Therefore in $] 0,2 M_{1}\left[, h\left(u, \chi_{r_{1}}(u)\right) \rightarrow h_{1}\left(r_{1}\right)\right.$ as $u \rightarrow \infty . h_{1}$ is a continuous function on $] 0,2 M_{1}[$ and the convergence is uniform in compact subintervals.

Integrating (1.13) along $\chi_{r_{1}}$ gives:

$$
\begin{gathered}
\frac{\partial h}{\partial r}\left(u_{1}, \chi_{r_{1}}\left(u_{1}\right)\right)=\exp \left[\int_{0}^{u_{1}}\left[\frac{(g-\bar{g})}{r}\right]_{\chi_{r_{1}}}(u) d u\right]\left\{\frac{\partial h}{\partial r}\left(0, \chi_{r_{1}}(0)\right)\right. \\
+\int_{0}^{u_{1}}\left[\frac{1}{2 r^{2}}\left(-3(g-\bar{g})+4 \pi g(h-\bar{h})^{2}(h-\bar{h})\right]_{\chi_{r_{1}}}(u) \exp \left[-\int_{0}^{u}\left[\frac{(g-\bar{g})}{r}\right]_{\chi_{r_{1}}}\left(u^{\prime}\right) d u^{\prime}\right] d u\right\} .
\end{gathered}
$$

Taking then into account (4.7), (4.8), and (4.9), we obtain that for all $u_{1} \geqq 0$ and $\left.r_{1} \in\right] 0,2 M_{1}[$

$$
\left|\frac{\partial h}{\partial r}\left(u_{1}, \chi_{r_{1}}(u)\right)\right| \leqq c_{1}^{\prime}\left(r_{1}\right)
$$


where

$$
\begin{aligned}
c_{1}^{\prime}\left(r_{1}\right):= & e^{\left(u_{0}\left(r_{1}\right)+32 M_{1}\right) / r_{1}}\left\{\left|\frac{\partial h}{\partial r}\left(0, \chi_{r_{1}}(0)\right)\right|\right. \\
& \left.+\frac{1}{2 r_{1}}\left(u_{0}\left(r_{1}\right)+32 M_{1}\right)\left[3+4 \pi\left(c_{1}\left(r_{1}\right)+c_{0}\left(r_{1}\right)\right)^{2}\right]\left(c_{1}\left(r_{1}\right)+c_{0}\left(r_{1}\right)\right)\right\},
\end{aligned}
$$

$c_{1}^{\prime}$ is a continuous function in the interval $] 0,2 M_{1}\left[\right.$. Then if $u_{2}>u_{1} \geqq u_{0}(r)$, taking into account (4.13), (4.9), (4.7), and (4.5), we obtain, in view of (1.13), that:

$$
\begin{aligned}
& \left|\frac{\partial h}{\partial r}\left(u_{2}, \chi_{r_{1}}\left(u_{2}\right)\right)-\frac{\partial h}{\partial r}\left(u_{1}, \chi_{r_{1}}\left(u_{1}\right)\right)\right| \leqq \int_{u_{1}}^{u_{2}}\left[\left|D\left(\frac{\partial h}{\partial r}\right)\right|\right]_{\chi_{r_{1}}}(u) d u \\
& \quad \leqq k^{\prime}\left(r_{1}\right)\left(e^{-u_{1} / 32 M_{1}}-e^{-u_{2} / 32 M_{1}}\right),
\end{aligned}
$$

where

$$
k^{\prime}\left(r_{1}\right):=\frac{16 M_{1}}{r_{1}^{2}} e^{u_{0}\left(r_{1}\right) / 32 M_{1}}\left\{2 r_{1} c_{1}^{\prime}\left(r_{1}\right)+\left[3+4 \pi\left(c_{1}\left(r_{1}\right)+c_{0}\left(r_{1}\right)\right)^{2}\right]\left(c_{1}\left(r_{1}\right)+c_{0}\left(r_{1}\right)\right)\right\} .
$$

Since $k^{\prime}$ is a continuous function on $] 0,2 M_{1}[$, (4.15) implies that in $] 0,2 M_{1}[$, $\partial h / \partial r\left(u, \chi_{r_{1}}(u)\right) \rightarrow h_{1}^{\prime}\left(r_{1}\right)$ as $u \rightarrow \infty, h_{1}^{\prime}$ is a continuous function on $] 0,2 M_{1}[$, and the convergence is uniform in compact subintervals. It follows easily that $h_{1}^{\prime}=\partial h_{1} / \partial r$.

By (3.26) for each $\left.r_{1} \in\right] 0,2 M_{1}\left[\right.$ and each $u_{1} \geqq u_{0}\left(r_{1}\right)$, we have

$$
\int_{u_{1}}^{\infty}[\bar{g}]_{\chi_{r_{1}}}(u) d u \leqq 32 M_{1} e^{-\left(u_{1}-u_{0}\left(r_{1}\right)\right) / 32 M_{1}} .
$$

It follows that the convergence of $\chi_{r_{1}}(u)$ to $r_{1}$ as $u \rightarrow \infty$ is uniform in any compact subinterval of $] 0,2 M_{1}[$. This fact together with the above implies that at each $\left.r_{1} \in\right] 0,2 M_{1}\left[, \bar{h}\left(u, r_{1}\right) \rightarrow \bar{h}_{1}\left(r_{1}\right), h\left(u, r_{1}\right) \rightarrow h_{1}\left(r_{1}\right), \partial h / \partial r\left(u, r_{1}\right) \rightarrow \partial h_{1} / \partial r\left(r_{1}\right)\right.$ as $u \rightarrow \infty$, and the convergence is uniform in any compact subinterval of the interval $] 0,2 M_{1}[$.

According to Proposition 3 of [3], for any generalized solution the quantity

$$
\int_{0}^{\infty} h^{2}(u, r) d r
$$

is an absolutely continuous function of $u$ and

$\frac{\partial}{\partial u}\left(\int_{0}^{\infty} h^{2}(u, r) d r\right)+\frac{1}{2} \int_{0}^{\infty}\left[(g-\bar{g}) h^{2}+\bar{g}(h-\bar{h})^{2}\right](u, r) \frac{d r}{r}+\frac{1}{2} f^{2}(u)=\frac{1}{8 \pi}(1-g(u, 0))$.

Here

$$
f(u)=\lim _{r \rightarrow \infty}\left(\bar{g}^{1 / 2} \bar{h}\right)(u, r),
$$

where, according to Proposition 2 of [3], the limit is defined for almost all $u$, and we have $f \in L^{2}\left(0, u_{0}\right), u_{0}$ arbitrary. On the other hand, the function

$$
\int_{r}^{\infty} h^{2} d r
$$


is continuously differentiable in the complement of the central line, and from the nonlinear evolution equation we deduce that

$$
D\left(\int_{r}^{\infty} h^{2} d r\right)=-\frac{1}{2} \int_{r}^{\infty}\left[(g-\bar{g}) \bar{h}^{2}+\bar{g}(h-\bar{h})^{2}\right] \frac{d r}{r}+\frac{1}{8 \pi}(1-g) .
$$

Therefore, the function

$$
\int_{0}^{r} h^{2} d r
$$

is weakly differentiable in the complement of the central line and, by (4.17) and (4.18),

$$
D\left(\int_{0}^{r} h^{2} d r\right)=-\frac{1}{2} \int_{0}^{r}\left[(g-\bar{g}) h^{2}+\bar{g}(h-\bar{h})^{2}\right] \frac{d r}{r}-\frac{1}{2} f^{2}(u)+\frac{1}{8 \pi}(g(u, r)-g(u, 0)) .
$$

Integrating (4.19) along a characteristic $\left.\chi_{r_{1}}, r_{1} \in\right] 0,2 M_{1}[$, we obtain:

$$
\begin{aligned}
& \int_{0}^{\chi_{r_{1}}\left(u_{1}\right)} h^{2}\left(u_{1}, r\right) d r+\frac{1}{2} \underset{Q\left(\chi_{r_{1}} ; u_{1}\right)}{ }\left[(g-\bar{g}) \bar{h}^{2}+\bar{g}(h-\bar{h})^{2}\right] \frac{d r}{r} d u \\
& +\frac{1}{2} \int_{0}^{u_{1}} f^{2}(u) d u+\frac{1}{8 \pi} \int_{0}^{u_{1}} g(u, 0) d u=\int_{0}^{\chi_{r_{1}}(0)} h^{2}(0, r) d r \\
& +\frac{1}{8 \pi} \int_{0}^{u_{1}}[g]_{\chi_{\nu_{1}}}(u) d u
\end{aligned}
$$

where

$$
Q\left(\chi_{r_{1}} ; u_{1}\right):=\left\{(u, r) \mid 0<u<u_{1}, 0<r<\chi_{r_{1}}(u)\right\}
$$

Considering (4.8) we conclude from (4.20) that for all $u \geqq 0$ :

$$
\int_{0}^{\chi_{r_{1}}(u)} h^{2}(u, r) d r<C\left(r_{1}\right)
$$

and therefore, a fortiori,

$$
\int_{0}^{r_{1}} h^{2}(u, r) d r \leqq C\left(r_{1}\right)
$$

Here

$$
C\left(r_{1}\right):=\int_{0}^{\chi_{r_{1}}(0)} h^{2}(0, r) d r+\frac{1}{8 \pi}\left(u_{0}\left(r_{1}\right)+32 M_{1}\right)
$$

Since $h(u, r)$ converges pointwise in $\left.] 0, r_{1}\right]$ to $h_{1}(r)$ as $u \rightarrow \infty,(4.22)$ implies by Fatou's lemma that $h_{1} \in L^{2}\left(0, r_{1}\right)$, and

$$
\int_{0}^{r_{1}} h_{1}^{2}(r) d r \leqq \liminf _{u \rightarrow \infty} \int_{0}^{r_{1}} h^{2}(u, r) d r .
$$


We shall finally show that $\bar{h}_{1}$ is indeed the mean value function of $h_{1}$. By the above for each $\delta$ and $r$ such that $0<\delta<r<2 M_{1}$, we have on one hand,

$$
\int_{\delta}^{r} h\left(u, r^{\prime}\right) d r^{\prime} \rightarrow \int_{\delta}^{r} h_{1}\left(r^{\prime}\right) d r^{\prime} \quad \text { as } \quad u \rightarrow \infty
$$

and, on the other hand,

$$
\int_{\delta}^{r} h\left(u, r^{\prime}\right) d r^{\prime}=r \bar{h}(u, r)-\delta \bar{h}(u, \delta) \rightarrow r \bar{h}_{1}(r)-\delta \bar{h}_{1}(\delta) \quad \text { as } \quad u \rightarrow \infty .
$$

Hence,

$$
r \bar{h}_{1}(r)=\delta \bar{h}_{1}(\delta)+\int_{\delta}^{r} h_{1}\left(r^{\prime}\right) d r^{\prime}
$$

From (4.22) we obtain:

$$
\delta|\bar{h}(u, \delta)| \leqq \int_{0}^{\delta}|h(u, r)| d r \leqq \delta^{1 / 2}\left(\int_{0}^{\delta} h^{2}(u, r) d r\right)^{1 / 2} \leqq \delta^{1 / 2}(C(\delta))^{1 / 2}
$$

Therefore

$$
\delta\left|\bar{h}_{1}(\delta)\right| \leqq \delta^{1 / 2}(C(\delta))^{1 / 2} \rightarrow 0 \quad \text { as } \quad \delta \rightarrow 0
$$

[since $C\left(r_{1}\right)$ is an increasing function of $r_{1}$ ]. Consequently letting $\delta \rightarrow 0$ in (4.24), we obtain [in view of the fact that $h_{1}$ is integrable on $\left.(0, r)\right]$ :

$$
\bar{h}_{1}(r)=\frac{1}{r} \int_{0}^{r} h_{1}\left(r^{\prime}\right) d r^{\prime},
$$

and the proof of Theorem 3 is complete.

We note that $h_{1} \notin L^{2}\left(0,2 M_{1}\right)$.

Letting $u_{1} \rightarrow \infty$ in (4.20), we deduce

Corollary 2. $f \in L^{2}(0, \infty)$.

We also deduce

Corollary 3. At each $\left.r_{1} \in\right] 0,2 M_{1}[$,

$$
(\bar{g} / g)\left(u, r_{1}\right) \rightarrow e^{-2 \lambda_{1}\left(r_{1}\right)} \quad \text { as } \quad u \rightarrow \infty,
$$

where

$$
e^{-2 \lambda_{1}\left(r_{1}\right)}:=\frac{1}{r_{1}} \int_{0}^{r_{1}} \exp \left[-4 \pi \int_{r}^{r_{1}}\left(h_{1}-\bar{h}_{1}\right)^{2}\left(r^{\prime}\right) \frac{d r^{\prime}}{r^{\prime}}\right] d r,
$$

and the convergence is uniform in any compact subinterval of $] 0,2 M_{1}[$. Also,

$$
e^{-2 \lambda_{1}\left(r_{1}\right)}=1-\frac{2 m_{1}\left(r_{1}\right)}{r_{1}} .
$$

Proof. Let $\left.r_{1} \in\right] 0,2 M_{1}[$. We have

$$
\left(\frac{\bar{g}}{g}\right)\left(u, r_{1}\right)=e^{-2 \lambda\left(u, r_{1}\right)}=\frac{1}{r_{1}} \int_{0}^{r_{1}} \exp \left[-4 \pi \int_{r}^{r_{1}}(h-\bar{h})^{2}\left(u, r^{\prime}\right) \frac{d r^{\prime}}{r^{\prime}}\right] d r .
$$


Consider the integral

$$
\alpha(u, r):=\int_{r}^{r_{1}}(h-\bar{h})^{2}\left(u, r^{\prime}\right) \frac{d r^{\prime}}{r^{\prime}} .
$$

By Theorem 3, for each $\varepsilon>0 \alpha(u, r) \rightarrow \alpha_{1}(r)$ as $u \rightarrow \infty$ uniformly in $\left[\varepsilon, r_{1}\right]$, where:

$$
\alpha_{1}(r):=\int_{r}^{r_{1}}\left(h_{1}-\bar{h}_{1}\right)^{2}\left(r^{\prime}\right) \frac{d r^{\prime}}{r^{\prime}} .
$$

Thus for every $\varepsilon, \delta>0$ there exists a $u_{2}(\varepsilon, \delta)$ such that $u>u_{2}(\varepsilon, \delta)$ implies

$$
\left|\alpha(u, r)-\alpha_{1}(r)\right|<\delta \text { for all } r \in\left[\varepsilon, r_{1}\right] \text {. }
$$

Given now any $\eta>0$, let us set

$$
u_{1}(\eta)=u_{2}\left(\eta r_{1} / 2, \eta / 8 \pi\right)
$$

Then $u>u_{2}(\eta)$ implies:

$$
\int_{\eta r_{1} / 2}^{r_{1}}\left|e^{-4 \pi \alpha(u, r)}-e^{-4 \pi \alpha_{1}(r)}\right| d r<\eta r_{1} / 2
$$

and, since

$$
\int_{0}^{\eta r_{1} / 2}\left|e^{-4 \pi \alpha(u, r)}-e^{-4 \pi \alpha_{1}(r)}\right| d r \leqq \eta r_{1} / 2
$$

$u>u_{2}(\eta)$ implies in fact that:

$$
\left|e^{-2 \lambda\left(u, r_{1}\right)}-e^{-2 \lambda_{1}\left(r_{1}\right)}\right|=\frac{1}{r_{1}}\left|\int_{0}^{r_{1}}\left(e^{-4 \pi \alpha(u, r)}-e^{-4 \pi \alpha_{1}(r)}\right) d r\right|<\eta .
$$

Thus $e^{-2 \lambda\left(u, r_{1}\right)} \rightarrow e^{-2 \lambda_{1}\left(r_{1}\right)}$ as $u \rightarrow \infty$. The fact that the convergence is uniform in any compact subinterval of $] 0,2 M_{1}[$ follows easily. Now

$$
e^{-2 \lambda\left(u, r_{1}\right)}=1-\frac{2 m\left(u, r_{1}\right)}{r_{1}},
$$

and by (4.2) $m\left(u, r_{1}\right) \rightarrow m_{1}\left(r_{1}\right)$ as $u \rightarrow \infty$. Hence

$$
e^{-2 \lambda_{1}\left(r_{1}\right)}=1-\frac{2 m_{1}\left(r_{1}\right)}{r_{1}} .
$$

For each $\left.r_{1} \in\right] 0,2 M_{1}[$, let us denote:

$$
Q\left(\chi_{r_{1}}\right):=\left\{(u, r) \mid u>0,0<r<\chi_{r_{1}}(u)\right\} .
$$

We finally have

Corollary 4. $e^{2 \lambda_{1}} \in L^{1}\left(0, r_{1}\right)$ and $g^{1 / 2} \xi / \bar{g} r^{1 / 2} \in L^{2}\left(Q\left(\chi_{r_{1}}\right)\right)$, each $r_{1}<2 M_{1}$.

Proof. The first conclusion is in fact equivalent to the conclusion of Theorem 3 that $h_{1} \in L^{2}\left(0, r_{1}\right)$ for each $r_{1}<2 M_{1}$, but we shall deduce it here directly from the main integral identity which yields also the second conclusion. Along the characteristic 
$\chi_{r_{1}}$ the main integral identity reads

$$
\int_{0}^{\chi_{r_{1}}\left(u_{1}\right)} e^{2 \lambda\left(u_{1}, r\right)} d r+2 \pi \iint_{Q\left(x_{r_{1}} ; u_{1}\right)} \frac{g \xi^{2}}{\bar{g}^{2} r} d r d u+\frac{1}{2} \int_{0}^{u_{1}} g(u, 0) d u=\int_{0}^{\chi_{r_{1}}(0)} e^{2 \lambda(0, r)} d r .
$$

Hence, for all $u \geqq 0$,

$$
\int_{0}^{r_{1}} e^{2 \lambda(u, r)} d r \leqq \int_{0}^{x_{r_{1}}(0)} e^{2 \lambda(0, r)} d r,
$$

and Corollary 2 implies that $e^{2 \lambda(u, r)}$ converges pointwise in $\left.] 0, \mathrm{r}_{1}\right]$ to $e^{2 \lambda_{1}(r)}$ as $u$ $\rightarrow \infty$. We conclude by Fatou's lemma that $e^{2 \lambda_{1}} \in L^{1}\left(0, r_{1}\right)$ and

$$
\int_{0}^{r_{1}} e^{2 \lambda_{1}(r)} d r \leqq \liminf _{u \rightarrow \infty} \int_{0}^{r_{1}} e^{2 \lambda(u, r)} d r .
$$

Letting $u_{1} \rightarrow \infty$ in (4.25), we deduce also that

$$
g^{1 / 2} \xi / \bar{g} r^{1 / 2} \in L^{2}\left(Q\left(\chi_{r_{1}}\right)\right) \text {. }
$$

We note that as $r_{1} \rightarrow 2 M_{1}, \chi_{r_{1}}(0) \rightarrow \infty$. The behaviour of the scalar field at the point at infinity on the horizon needs further investigation.

\section{References}

1. Christodoulou, D.: The problem of a self-gravitating scalar field. Commun. Math. Phys. 105, 337 (1986)

2. Christodoulou, D.: Global existence of generalized solutions of the spherically symmetric Einstein-scalar equations in the large. Commun. Math. Phys. 106, 587 (1986)

3. Christodoulou, D.: The structure and uniqueness of generalized solutions of the spherically symmetric Einstein-scalar equations. Commun. Math. Phys. 109, 591 (1987)

Communicated by S.-T. Yau

Received November 9, 1984; in revised form August 29, 1986 
